\title{
Mean-field approximation of network of biophysical neurons driven by conductance-based ion exchange
}

\author{
Abhirup Bandyopadhyay ${ }^{1}$, Christophe Bernard ${ }^{1}$, Viktor K. Jirsa ${ }^{1 *}$, and Spase Petkoski ${ }^{1 *}$ \\ ${ }^{1}$ Aix Marseille University, INSERM, Institute de Neuroscience des Systems, Marseille, 13005 France \\ *e-mail: viktor.jirsa@univ-amu.fr, spase.petkoski@univ-amu.fr \\ *These authors contributed equally to this work as last author
}

\begin{abstract}
Numerous network and whole brain modeling approaches make use of mean-field models. Their relative simplicity allows studying network dynamics at a large scale. They correspond to lumped descriptions of neuronal assemblies connected via synapses. mean-field models do not consider the ionic composition of the extracellular space, which can change in physiological and pathological conditions, with strong effects on neuron activity. Here we derive a mean-field model of a population of Hodgkin-Huxley type neurons, which links the neuronal intra- and extra-cellular ion concentrations to the mean membrane potential and the mean synaptic input in terms of the synaptic conductance. The model can generate various physiological brain activities including multi-stability at resting states, as well as pathological spiking and bursting behaviors, and depolarization block. The results from the analytical solution of the mean-field model agree with the mean behavior of numerical simulations of large-scale networks of neurons. The mean-field model is analytically exact for non-autonomous ion concentration variables and provides a mean-field approximation in the thermodynamic limit, for locally homogeneous mesoscopic networks of biophysical neurons driven by an ion-exchange mechanism. These results may provide the missing link between high-level neural mass approaches which are used in the brain network modeling and physiological parameters that drive the neuronal dynamics.
\end{abstract}

Key words: Hodgkin-Huxley type neurons, Mean-field, Resting state, Epilepsy, Lorentzian ansatz

\begin{abstract}
Significance Statement: In this study, we applied mathematical formalism to estimate the mean-field behaviors of a large neuronal ensemble taking into account the ion-exchange between the intracellular and extracellular space. The model demonstrates different brain activities including resting state, spiking behavior, and seizure, as a function of the extracellular ion concentration. The relevant parameter regime for different brain activities is extracted mainly in terms of ion concentration and heterogeneity of individual neurons in the network. This neural mass model enables studying the influence of changes in extracellular ionic conditions on whole brain dynamics in health and disease. The effect of external stimulus current and conductance-based coupling of neural masses are also analyzed.
\end{abstract}

\section{Introduction}

Large-scale brain dynamics can be studied in silico with network models (Deco et al., 2011). Local activity can be represented by neuronal mass models (Deco et al., 2008), which coupled together through synapses, time delays and noise (Deco and Jirsa, 2012, Petkoski and Jirsa, 2019) allow the emergence of whole brain activity that can be linked to empirical neuroimaging data (Sanz-Leon et al., 2015). The observable properties (variables) in the population level of a large-scale ensemble are generally explained by statistical physics formalism of mean-field theory (Wilson and Cowan, 1972, David and Friston, 2003, Moran et al., 2007, Wong and Wang, 2006). The models demonstrated a predictive value for resting state activity (Melozzi et al., 2019, Courtiol et al., 2020), or for seizure genesis and propagation in epilepsy (Proix et al., 2017). Neural mass models have a low enough number of parameters to be tractable and provide general intuitions regarding mechanisms (Jirsa et al., 2014, Jirsa et al., 2017, Deco et al., 2021, Amunts et al., 2020). Although it is not practical to include all known biophysical parameters, it may be important to include parameters that can have widespread and general effects on neuronal activity (Amunts et al., 2020).

The concentration of $\mathrm{Na}^{+}, \mathrm{K}^{+}, \mathrm{Ca}^{2+}$, and $\mathrm{Cl}^{-}$ions in the extracellular space is a key parameter to consider. Extracellular ion concentrations change dynamically in vivo as a function of the brain state, for example between arousal and sleep (Ding et 
al., 2016). Changes in extracellular ion concentrations can switch one brain state to another (Ding et al., 2016). Extracellular potassium concentration $\left(\left[\mathrm{K}^{+}\right]_{o}\right)$ plays a central role. Transient changes in $\left(\left[\mathrm{K}^{+}\right]_{o}\right)$ can have large effects on cell excitability and spontaneous neuronal activity (Amzica et al., 2002, Ransom et al., 2000, Cressman et al., 2009), a result consistently reported in modeling studies (Bazhenov et al., 2004, Park and Durand, 2006, Fröhlich et al., 2008). Increases in $\left[\mathrm{K}^{+}\right]_{o}$ are tightly controlled by astrocytes, which can efficiently pump $\left[K^{+}\right]_{o}$ and distribute it via their syncytium to prevent hyperexcitability (Breslin et al., 2018, Nwaobi et al., 2016, Crunelli et al., 2015, Hansson et al., 2000, Bedner and Steinhäuser, 2013). Saturation or lack of efficiency of this buffering mechanisms is often linked to a pathological state. Detailed single neuron models demonstrate that continuous increases in $\left[\mathrm{K}^{+}\right]_{o}$ can lead to different firing states, from tonic, to bursting, to seizure-like events and depolarization block (Cressman et al., 2009, Depannemaecker et al., 2021). In such detailed models, the buffering action of astrocytes is represented by a parameter named $\left[K^{+}\right]_{\text {bath }}$ (Cressman et al., 2009, Breslin et al., 2018, Ullah et al., 2009, Nwaobi et al., 2016, Depannemaecker et al., 2021). Our goal is to use a similar approach but at the neural mass model level, to be incorporated to study whole brain dynamics.

Phenomenological neuron models have been studied to understand the macroscopic dynamics of neuronal populations (Izhikevich and Edelman, 2008) or to provide statistical descriptions of neuronal networks (Wilson and Cowan, 1972, Deco et al., 2008, Montbrió et al., 2015, Montbrió and Pazó, 2020). Statistical population measures, such as the firing rate, can be used to assess macroscopic dynamics (Shriki et al., 2003, Deco et al., 2011, Roxin et al., 2005, Luke et al., 2013, Jirsa et al., 2014, Proix et al., 2014, Sanz-Leon et al., 2015, Wendling et al., 2016, Ashwin et al., 2016). Recent studies combine local neuronal dynamics with statistical descriptions in terms of the mean membrane potential and the firing rate in quadratic integrate-and-fire (QIF) neurons (Montbrió et al., 2015, Devalle et al., 2018, Montbrió and Pazó, 2020). Neural mass models and large scale brain dynamics models are also used for analyzing and identification of chaos in brain signal (Baladron et al., 2012, Bandyopadhyay and Kar, 2018), epileptic seizure transmission (Spiegler et al., 2011, Touboul et al., 2011, Nevado-Holgado et al., 2012, Hocepied et al., 2013, El Houssaini et al., 2015a), phase oscillation (Laing, 2017, Byrne et al., 2017, Petkoski and Stefanovska, 2012), evolution of network synchrony (Bandyopadhyay and Kar, 2018, Petkoski and Stefanovska, 2012) and several other problems. Here we approximate ion-exchange dynamics by a step-wise QIF model with two slow timescale biophysical variables. We demonstrate that the distribution of the neurons' membrane potentials can be described by a Lorentzian ansatz (LA). The continuity equation is solved to give rise to a mean-field model with the same probability distribution of membrane potentials as the LA. The mean-field model is exact for non-autonomous ion concentration variables and provides a mean-field approximation within the thermodynamic limit, i.e., for a locally homogeneous mesoscopic network. We thus demonstrate a mean-field approximation for an all-to-all coupled network of heterogeneous neurons to approximately capture the behavior of ion-exchange-driven neuronal dynamics. Considering different distributions of heterogeneous input current, we obtain a mean-field approximation, described as a set of ordinary differential equations that fully described the macroscopic states of the recurrently connected spiking neurons, in various dynamical regimes that can be linked to different healthy and pathological states.

\section{Materials and Methods}

\section{Single neuron model}

The membrane potential of a single neuron in the brain is generally driven by an ion-exchange mechanism in intracellular and extracellular space. The concentrations of potassium, sodium, and chlorine in the intracellular and extracellular space along with the active transport pump $\left(\mathrm{Na}^{+} / \mathrm{K}^{+}\right.$pump) in the cell membrane of neurons generate input currents to a neuron cell that drive the electrical behavior of a single neuron in terms of its membrane potential. The ion-exchange mechanism in the cellular microenvironment, including local diffusion, glial buffering, ion pumps, and ion channels, has been mathematically modeled based on conductance-based ion dynamics to reflect the resting state and seizure behaviors in single neurons (Hodgkin and Huxley, 1952, Cressman et al., 2009, Ullah et al., 2009, Depannemaecker et al., 2021). The mechanism of ion-exchange in the intracellular and extracellular space of the neuronal membrane is represented schematically in Fig. 1. This biophysical interaction and ion-exchange mechanism across the membrane of a neuron cell can be described as a Hodgkin-Huxley type dynamical process, represented by the following dynamical system.

$$
\begin{aligned}
\frac{d V}{d t} & =-\frac{1}{C_{m}}\left(J_{C l}+J_{N a}+J_{K}+J_{\text {pump }}\right) \\
\frac{d n}{d t} & =\frac{n_{\infty}(V)-n}{\tau_{n}} \\
\frac{d \Delta\left[K^{+}\right]_{i}}{d t} & =-\frac{\gamma}{w_{i}}\left(J_{k}-2 J_{\text {pump }}\right) \\
\frac{d\left[K^{+}\right]_{g}}{d t} & =\varepsilon\left(\left[K^{+}\right]_{\text {bath }}+\beta \Delta\left[K^{+}\right]_{i}-\left\{\left[K^{+}\right]_{0, o}+\left[K^{+}\right]_{g}\right\}\right)
\end{aligned}
$$




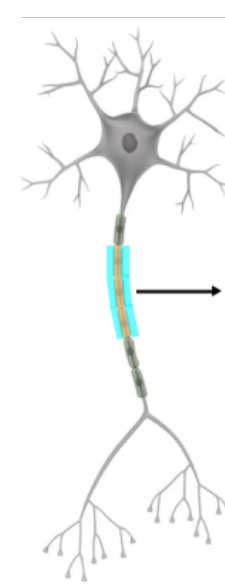

(a)

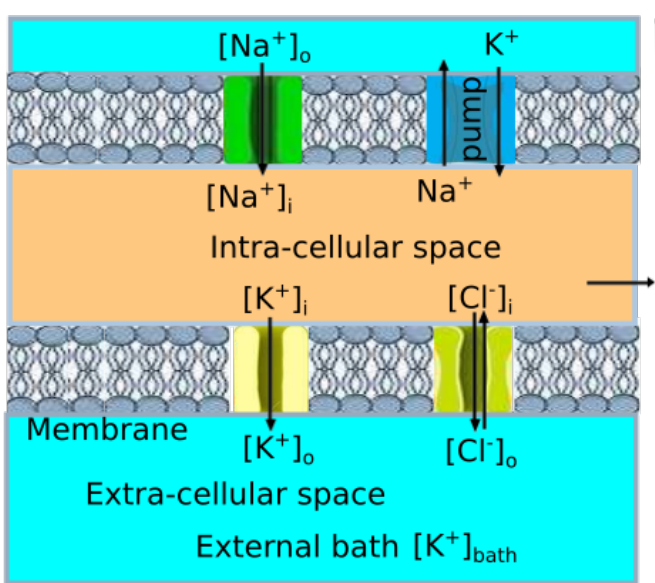

(b)

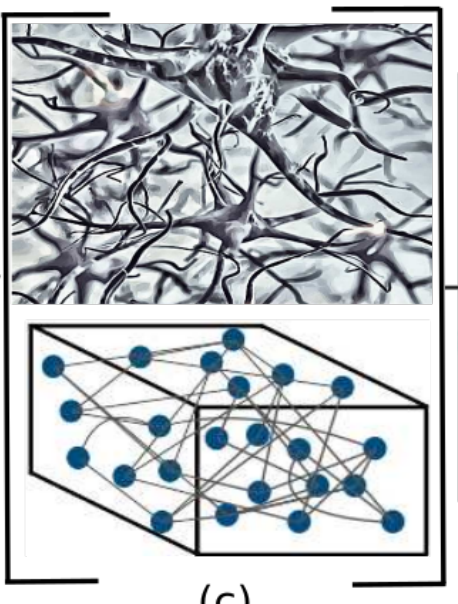

(c)

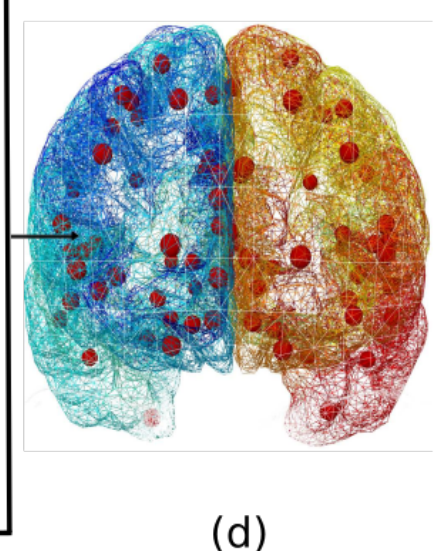

(d)

Figure 1. Schematic diagram of the ion channel mechanism in extracellular and intracellular space in the brain: (a) a single neuron highlighting intracellular (yellow) and extracellular (cyan) space, (shown in the soma for simplicity of drawing). (b) ion-exchange mechanism through the membrane in the cellular space: $\mathrm{Na}^{+}$gets inside the intra-cellular space and $\mathrm{K}^{+}$gets out, flow of $\mathrm{Cl}^{-}$is bidirectional; for the pump, $\mathrm{Na}^{+}$get out and $\mathrm{K}^{+}$gets into the intra-cellular space, and the cell membrane is shown by gray. (c) A population of interacting neurons (top) and point neurons (bottom). (d) Brain network model with activity of each brain region (centers of masses shown by red circles) represented by neural masses.

This model represents the ion-exchange mechanism of a single conductance-based neuron in terms of membrane potential $(V)$, the potassium conductance gating variable $(n)$, intracellular potassium concentration variation $\left(\Delta\left[K^{+}\right]_{i}\right)$ and extracellular potassium buffering by the external bath $\left(\left[K^{+}\right]_{g}\right)$. This mechanism considers ion-exchange through the sodium, potassium, calcium-gated potassium, intracellular sodium, and extracellular potassium concentration gradients and leak currents. The intrinsic ionic currents of the neuron along with the sodium-potassium pump current and potassium diffusion regulate the concentrations of the different ion concentrations. The Nernst equation was used to couple the membrane potential of the neuron with the concentrations of the ionic currents. This mechanism gives rise to a slow-fast dynamical system in which the membrane potential $(V)$ and potassium conductance gating variable $(n)$ constitute the fast subsystem and the slow subsystem is represented in terms of the variation in the intracellular potassium concentration $\left(\Delta\left[K^{+}\right]_{i}\right)$ and extracellular potassium buffering by the external bath $\left(\left[\mathrm{K}^{+}\right]_{g}\right.$ ) (in Eq. (1)); where input currents due to different ionic substances and pump are represented as follows (Cressman et al., 2009, Depannemaecker et al., 2021):

$$
\begin{aligned}
J_{N a} & =\left(g_{N a, l}+g_{N a} m_{\infty}(V) h(n)\right)\left(V-26.64 \log \left(\frac{\left[N a^{+}\right]_{o}}{\left[N a^{+}\right]_{i}}\right)\right) \\
J_{K} & =\left(g_{K, l}+g_{K} n\right)\left(V-26.64 \log \left(\frac{\left[K^{+}\right]_{o}}{\left[K^{+}\right]_{i}}\right)\right) \\
J_{C l} & =g_{C l}\left(V+26.64 \log \left(\frac{\left[C l^{-}\right]_{o}}{\left[C l^{-}\right]_{i}}\right)\right) \\
J_{\text {pump }} & =\rho \frac{1}{1+\exp \left(\frac{21-\left[\mathrm{Na}^{+}\right]_{i}}{2}\right)} \frac{1}{1+\exp \left(5.5-\left[\mathrm{K}^{+}\right]_{o}\right)}
\end{aligned}
$$

The conductance functions are represented as follows:

$$
\begin{aligned}
n_{\infty}(V) & =\frac{1}{1+\exp \left(\frac{-19-V}{18}\right)} \\
m_{\infty}(V) & =\frac{1}{1+\exp \left(\frac{-24-V}{12}\right)} \\
h(n) & =1.1-\frac{1}{1+\exp (-8(n-0.4))}
\end{aligned}
$$

In this model the concentration of chloride ion is invariant and the extracellular and intracellular concentrations of potassium, 
sodium, and chlorine ions are represented in terms of these state variables as follows:

$$
\begin{aligned}
\Delta\left[\mathrm{Na}^{+}\right]_{i} & =-\Delta\left[\mathrm{K}^{+}\right]_{i} \\
\Delta\left[\mathrm{Na}^{+}\right]_{o} & =-\beta \Delta\left[\mathrm{Na}^{+}\right]_{i} \\
\Delta\left[\mathrm{K}^{+}\right]_{o} & =-\beta \Delta\left[\mathrm{K}^{+}\right]_{i} \\
{\left[\mathrm{Na}^{+}\right]_{i} } & =\left[\mathrm{Na}^{+}\right]_{0, i}+\Delta\left[\mathrm{Na}^{+}\right]_{i} \\
{\left[\mathrm{Na}^{+}\right]_{o} } & =\left[\mathrm{Na}^{+}\right]_{0, o}+\Delta\left[\mathrm{Na}^{+}\right]_{o} \\
{\left[\mathrm{~K}^{+}\right]_{o} } & =\left[\mathrm{K}^{+}\right]_{0, o}+\Delta\left[\mathrm{K}^{+}\right]_{o}+[\mathrm{K}]_{g} \\
{\left[\mathrm{Cl}^{-}\right]_{o} } & =\left[\mathrm{Cl}^{-}\right]_{0, o},\left[\mathrm{Cl}^{-}\right]_{i}=\left[\mathrm{Cl}^{-}\right]_{0, i}
\end{aligned}
$$

The biophysically relevant values of the parameters could be obtained from several previous studies and from in vivo and in vitro experiments (Cressman et al., 2009, Depannemaecker et al., 2021). Those that we used for the simulation are shown in Table-1.

\begin{tabular}{|c|c|c|}
\hline Parameters & symbols & values \\
\hline Membrane capacitance & $C_{m}$ & $1 \mathrm{nF}$ \\
Gating time constant & $\tau_{n}$ & $4 S^{-1}$ \\
Chloride conductance & $g_{C l}$ & $7.5 \mathrm{nS}$ \\
Maximal potassium conductance & $g_{K}$ & $22 \mathrm{nS}$ \\
Maximal sodium conductance & $g_{N a}$ & $40 \mathrm{nS}$ \\
Potassium leak conductance & $g_{K, l}$ & $0.12 \mathrm{nS}$ \\
Sodium leak conductance & $g_{N a, l}$ & $0.02 \mathrm{nS}$ \\
Intracellular volume & $\omega_{i}$ & $2160 \mu \mathrm{m}$ \\
Extracellular volume & $\omega_{o}$ & $720 \mu \mathrm{m}$ \\
Intra/extra cellular volume ratio & $\beta=\frac{\omega_{i}}{\omega_{o}}$ & 3 \\
Concentration changes time constant & $\gamma$ & $0.04 S^{-1}$ \\
Diffusion time constant & $\varepsilon$ & $0.001 S^{-1}$ \\
Maximal Na/K pump current & $\rho$ & $250 \mathrm{pA}$ \\
External bath of K & {$\left[\mathrm{K}^{+}\right]_{b a t h}$} & $8 \mathrm{mM}$ \\
Initial concentration of Extracellular $\mathrm{K}$ & {$\left[\mathrm{K}^{+}\right]_{0, o}$} & $4.8 \mathrm{mM}$ \\
Initial concentration of Intracellular $\mathrm{K}$ & {$\left[\mathrm{K}^{+}\right]_{0, i}$} & $130 \mathrm{mM}$ \\
Initial concentration of Extracellular $\mathrm{Na}$ & {$\left[\mathrm{Na}^{+}\right]_{0, o}$} & $138 \mathrm{mM}$ \\
Initial concentration of Intracellular $\mathrm{Na}$ & {$\left[\mathrm{Na}^{+}\right]_{0, i}$} & $16 \mathrm{mM}$ \\
concentration of Extracellular $\mathrm{Cl}$ & {$\left[\mathrm{Cl}^{-}\right]_{0, o}$} & $112 \mathrm{mM}$ \\
concentration of Intracellular Cl & {$\left[\mathrm{Cl}^{-}\right]_{0, i}$} & $5 \mathrm{mM}$ \\
\hline
\end{tabular}

Table 1. List of parameters and their values used for the simulation

\section{Mean-field approximation of coupled neurons}

The next aim was to develop a mean-field model for a heterogeneous population of the all-to-all coupled biophysical neurons described by Eq. (1) within the thermodynamic limit, i.e., when the number of neurons $N \rightarrow \infty$. To consider the impact of other spiking neurons in a network on a single neuron through the synaptic input current, first the synaptic kinetics needed to be modeled. The basic mechanism of synaptic transmission can be described as the mechanism by which a neurotransmitter is released into the synaptic cleft as a result of an influx of calcium through presynaptic calcium channels as the presynaptic pulse (spike) depolarizes the synaptic terminal. The neurotransmitter plays a crucial role in opening the postsynaptic channels, which causes the flow of the ionic current across the membrane. This mechanism is often represented by phenomenological models, which assume that the normalized synaptic conductance $g_{s y n}(t)$ from 0 to 1 rises instantaneously at the time of the kth pulse (spike), $t^{k}$ and consequently undergoes an exponential decay with some rate constant $\tau$. This mechanism is traditionally modeled by the following differential equation

$$
\tau \frac{d g_{s y n}(t)}{d t}=\sum_{j=1}^{N}\left[-g_{s y n}(t)+\delta\left(t_{j}^{k}-t\right)\left(1-g_{s y n}(t)\right)\right]
$$


The solution can be written as $g_{s y n}(t)=\sum_{j=1}^{N}\left[\frac{t-t_{j}^{k}}{\tau} e^{\frac{t-t_{j}^{k}}{\tau}}\right]$ (Destexhe et al., 1994, Baroni et al., 2014). Here, $\delta(t)$ is the Dirac delta function, and $t_{j}^{k}$ represents the time of the kth pulse (spike) of the jth neuron. Then, the synaptic input current is given as follows:

$$
I_{\text {syn }}=k g_{\text {syn }}(t)(V-E)
$$

where $V$ is the postsynaptic potential; $E$ is the potential, termed the synaptic reversal potential, at which the direction of the net current flow reverses; and represents the maximum conductance of the synapse. At this point we approximated the four-dimensional dynamics in Eq. (1) to a three-dimensional system in order to represent the fast subsystem in terms of membrane potential alone. This allowed us to represent the probability distribution of $V$ in terms of a single fast variable $V$ and to use consequent mathematical formalism to solve the mean-field behavior of the large network of neurons. This was done by eliminating the second fast variable from the system by averaging $\frac{d V}{d t}$ over $n$, i.e., replacing $\frac{d V}{d t}=f\left(V, n, \Delta\left[K^{+}\right]_{i},\left[K^{+}\right]_{g}\right)$ with $\frac{d V}{d t}=\frac{1}{\limsup n-\liminf n} \int_{\liminf n}^{\limsup n} f\left(V, n, \Delta\left[K^{+}\right]_{i},\left[K^{+}\right]_{g}\right) d n$, where $f\left(V, n, \Delta\left[K^{+}\right]_{i},\left[K^{+}\right]_{g}\right)$ represents the right-hand side of the $V$ dynamics in Eq. (1). We modeled the average of $n$ as

$$
\langle n\rangle=\left\{\begin{array}{l}
n_{\infty}(V), \Delta\left[K^{+}\right]_{i}>\alpha \\
2.0+0.02\langle V\rangle, \Delta\left[K^{+}\right]_{i} \leq \alpha
\end{array}\right.
$$

where $\alpha=k_{\alpha}+\mu_{1}\left(\left[K^{+}\right]_{b a t h}-k_{0}\right)+\mu_{2}\left(\left[K^{+}\right]_{\text {bath }}-k_{0}\right)^{2}$ with $k_{\alpha}=-0.8825, \mu_{1}=-0.3965, \mu_{2}=0.0075, k_{0}=11.5$. Applying this averaging method and substituting $\langle n\rangle$ into the current terms

$$
\begin{aligned}
I_{N a} & =\left(g_{N a, l}+g_{N a} m_{\infty}(V) h(\langle n\rangle)\right)\left(V-26.64 \log \left(\frac{\left[N a^{+}\right]_{o}}{\left[N a^{+}\right]_{i}}\right)\right) \\
I_{K} & =\left(g_{K, l}+g_{K}\langle n\rangle\right)\left(V-26.64 \log \left(\frac{\left[K^{+}\right]_{o}}{\left[K^{+}\right]_{i}}\right)\right) \\
I_{C l} & =J_{C l}=g_{C l}\left(V+26.64 \log \left(\frac{\left[C l^{-}\right]_{o}}{\left[C l^{-}\right]_{i}}\right)\right) \\
I_{\text {pump }} & =J_{\text {pump }}=\rho \frac{1}{1+\exp \left(\frac{21-\left[N a^{+}\right]_{i}}{2}\right)} \frac{1}{1+\exp \left(5.5-\left[K^{+}\right]_{o}\right)}
\end{aligned}
$$

we obtained the 3-dimensional averaged dynamical model for Eq. (1) as follows

$$
\begin{aligned}
\frac{d V}{d t} & =-\frac{1}{C_{m}}\left(I_{C l}+I_{N a}+I_{K}+I_{\text {pump }}\right) \\
\frac{d \Delta\left[K^{+}\right]_{i}}{d t} & =-\frac{\gamma}{w_{i}}\left(I_{k}-2 I_{\text {pump }}\right) \\
\frac{d\left[K^{+}\right]_{g}}{d t} & =\varepsilon\left(\left[K^{+}\right]_{\text {bath }}+\beta \Delta\left[K^{+}\right]_{i}-\left\{\left[K^{+}\right]_{0, o}+\left[K^{+}\right]_{g}\right\}\right)
\end{aligned}
$$

In this way we obtained the system of the following set of ordinary differential equations to describe the microscopic population state of the ion-exchange driven network of biophysical neurons:

$$
\begin{aligned}
\frac{d V_{j}}{d t} & =-\frac{1}{C_{m}}\left(I_{C l}^{j}+I_{N a}^{j}+I_{K}^{j}+I_{\text {pump }}^{j}\right)+J g_{\text {syn }}(t)\left(V_{j}-E_{j}\right)+\eta_{j} ; \quad j=1,2, \ldots, N \\
\frac{d \Delta\left[K^{+}\right]_{i}}{d t} & =-\frac{\gamma}{w_{i}}\left(I_{k}-2 I_{\text {pump }}\right) \\
\frac{d\left[K^{+}\right]_{g}}{d t} & =\varepsilon\left(\left[K^{+}\right]_{\text {bath }}+\beta \Delta\left[K^{+}\right]_{i}-\left\{\left[K^{+}\right]_{0, o}+\left[K^{+}\right]_{g}\right\}\right)
\end{aligned}
$$

where $\mathrm{J}$ is the global coupling coefficient, $\eta_{j}$ is the heterogeneous quenched external input, and $E_{j}$ is the synaptic reversal potential of jth neuron. For the thermodynamic limit $N \rightarrow \infty$, is the heterogeneous quenched external input could be considered as a random variable $\eta$ which reduces Eq. (11) to the following form

$$
\begin{aligned}
\frac{d V}{d t} & =-\frac{1}{C_{m}}\left(I_{C l}+I_{N a}+I_{K}+I_{\text {pump }}\right)+J g_{\text {syn }}(t)(V-E)+\eta \\
\frac{d \Delta\left[K^{+}\right]_{i}}{d t} & =-\frac{\gamma}{w_{i}}\left(I_{k}-2 I_{\text {pump }}\right) \\
\frac{d\left[K^{+}\right]_{g}}{d t} & =\varepsilon\left(\left[K^{+}\right]_{\text {bath }}+\beta \Delta\left[K^{+}\right]_{i}-\left\{\left[K^{+}\right]_{0, o}+\left[K^{+}\right]_{g}\right\}\right)
\end{aligned}
$$


The $\frac{d V}{d t}$ equation in Eq. (12) is modeled as a step wise quadratic function based on the nullcline geometry of the microscopic state of the membrane potential $V$ and the dynamics of the biophysical population of neurons $\left\{V_{j}\right\}: j=1,2, \ldots, N$ is hence represented as the following step wise quadratic function of $\left\{V_{j}\right\}$.

$$
\frac{d V_{j}}{d t}=\left\{\begin{array}{l}
b_{1}\left\{\left(V_{j}-d_{1, j}\right)^{2}+I_{1, j}+J g_{s y n}(t)\left(V_{j}-E_{j}\right)\right\} ; \forall V_{j} \geq s \\
b_{2}\left\{\left(V_{j}-d_{2, j}\right)^{2}+I_{2, j}+J g_{s y n}(t)\left(V_{j}-E_{j}\right)\right\} ; \forall V_{j}<s
\end{array} \quad ; \quad j=1,2, \ldots, N\right.
$$

For a homogeneous population within the thermodynamic limit $N \rightarrow \infty$ this can be represented by removing the index as follows:

$$
\frac{d V}{d t}=\left\{\begin{array}{l}
b_{1}\left\{\left(V-d_{1}\right)^{2}+I_{1}+J g_{\text {syn }}(t)(V-E)\right\} ; \forall V \geq s \\
b_{2}\left\{\left(V-d_{2}\right)^{2}+I_{2}+J g_{s y n}(t)(V-E)\right\} ; \forall V<s
\end{array}\right.
$$

Here the terms $I_{1}$ and $I_{2}$ are functions of $\Delta\left[K^{+}\right]_{i}$ and $\left[K^{+}\right]_{\text {bath }}$ but are independent of $\mathrm{V}$ and are given as follows:

$$
\begin{aligned}
& I_{1}=\frac{\psi_{1}}{b_{1}}\left(\Delta\left[K^{+}\right]_{i}-k_{0}\right)+\frac{a_{1}}{b_{1}}+\eta, \\
& I_{2}=\frac{\psi_{2}}{b_{2}}\left(\Delta\left[K^{+}\right]_{i}-k_{0}\right)+\frac{a_{2}}{b_{2}}+\eta
\end{aligned}
$$

Here $d_{1}, d_{2}$ and $a_{2}$ are functions of $\left[K^{+}\right]_{b a t h}$ and $a_{1}$ which, in turn, is function of $\Delta\left[K^{+}\right]_{i}$ and $\left[K^{+}\right]_{b a t h}$. The parameters $b_{1}, b_{2}$, and $s$, are constants estimated from the nullclines geometry of system Eq. (1) as follows:

$$
\begin{aligned}
& d_{1}=q_{1}+r_{11}\left(\left[K^{+}\right]_{\text {bath }}-k_{0}\right)++r_{12}\left(\left[K^{+}\right]_{\text {bath }}-k_{0}\right)^{2}, \\
& d_{2}=q_{2}+r_{21}\left(\left[K^{+}\right]_{\text {bath }}-k_{0}\right)+r_{22}\left(\left[K^{+}\right]_{\text {bath }}-k_{0}\right)^{2}, \\
& a_{2}=q_{3}+\lambda_{1}\left(\left[K^{+}\right]_{\text {bath }}-k_{0}\right)+\lambda_{2}\left(\left[K^{+}\right]_{\text {bath }}-k_{0}\right)^{2}, \\
& a_{1}=a_{2}+\left(\psi_{2}-\psi_{1}\right)\left(\Delta\left[K^{+}\right]_{i}-k_{0}\right)-b_{1}\left(s-d_{1}\right)^{2}+b_{2}\left(s-d_{2}\right)^{2}
\end{aligned}
$$

with the following parameter values $s=-37, b_{1}=-0.50, b_{2}=0.11, k_{0}=11.5, r_{11}=0.45, r_{12}=-0.50, r_{21}=2.5, r_{22}=$ $-0.1, \lambda_{1}=30.0, \lambda_{2}=-0.05, q_{1}=-25.2, q_{2}=-56.0, q_{3}=-72.5, \psi_{1}=50.0, \psi_{2}=112.5$.

The validity of the fit of these parameters is shown in Fig. 2.

Also, $\eta$ in the Eq. (14) refers to the heterogeneous quenched component, which represents the heterogeneity in the network of neurons and is distributed according to some probability distribution, say $g(\eta)$. For the mean-field approximation in the thermodynamic limit $N \rightarrow \infty$, let us denote $\rho\left(V, \Delta\left[K^{+}\right]_{i},\left[K^{+}\right]_{b a t h}, \eta, t\right) d V$ as the fraction of neurons with a membrane potential between $V$ and $V+d V$ where $\eta$ is the random variable heterogeneity parameter (heterogeneous input current), which could be considered to be distributed according to the probability distribution $g(\eta)$. Then, the total voltage density can be written as $\int_{-\infty}^{\infty} \rho\left(V, \Delta\left[K^{+}\right]_{i},\left[K^{+}\right]_{b a t h}, \eta, t\right) g(\eta) d \eta$. This setup leads to the continuity equation

$$
\delta_{t} \rho+\delta_{V}\left[\frac{d V}{d t} \rho\right]=0
$$

It should be noted here that the approximation of Eq. (1) by Eq. (10) by removing $n$ from the system through the averaging method is an intermediate approximation, which ensures the integrability of the $\rho$ and allowed us to solve the continuity equation Eq. (15). However, we reused the functional forms in the mean-field equation as in Eq. (1) and continued this through the solution of the continuity equation. This resulted in retaining the original dynamic behavior of the system, and the intermediate approximation allowed us to derive the mean-field equation analytically. At this point adiabatic reduction was applied to the slow variables, and the differential equation representing the $V$ dynamics along with the coupling term was considered to be involved with only the $V, \eta$, and $t$ variables. This reduced the $V$ dynamics ( $\frac{d V}{d t}$ equation) in Eq. (12) to a function of $V$ and $t$ only for each value of $\eta$. Consequently, we could consider $\rho$ as a function of only $V, \eta$, and $t$ (as similar methodologies were applied in different literature like (Kuramoto, 1991, Petkoski and Stefanovska, 2012)). Therefore, we denoted the right-hand side of the $V$ dynamics as a function $f(t, V, \eta)$ and represent it as follows:

$\frac{d V}{d t}=-\frac{1}{C_{m}}\left(I_{C l}+I_{N a}+I_{K}+I_{\text {pump }}\right)+J g_{s y n}(t)(V-E)+\eta=f(t, V, \eta)$

That reduces Eq. (15) to $\frac{\delta \rho}{\delta t}+f(t, V) \frac{\delta \rho}{\delta V}=-f_{V}(t, V) \rho$ which is a first order quasi-linear PDE that can be solved by the Lagrange method using the Lagrange subsidiary equation

$$
\frac{d t}{1}=\frac{d V}{f(t, V)}=\frac{d \rho}{-f_{V}(t, V) \rho}
$$


bioRxiv preprint doi: https://doi.org/10.1101/2021.10.29.466427; this version posted December 7, 2021. The copyright holder for this preprint (which was not certified by peer review) is the author/funder, who has granted bioRxiv a license to display the preprint in perpetuity. It is made Biophysical neural mass model of ion-exchange available under aCC-BY-NC-ND 4.0 International license.

(a)

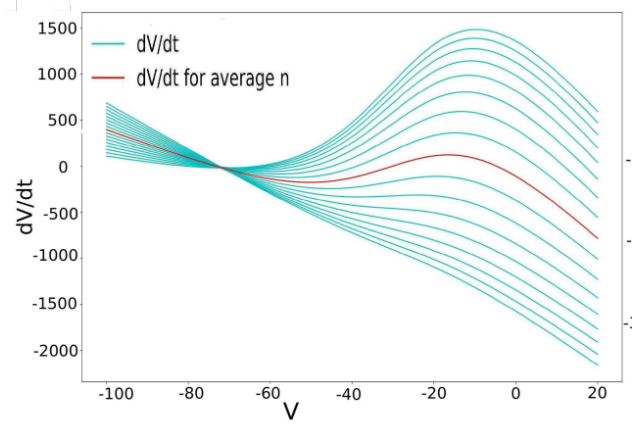

(d) $\left[K^{+}\right]_{\text {bath }}=6.5$

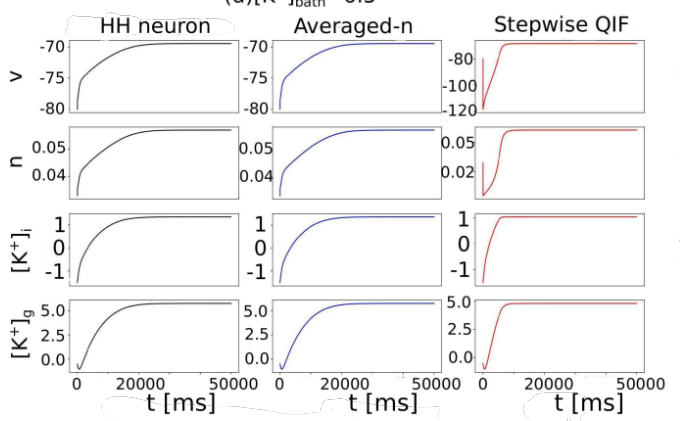

(b)

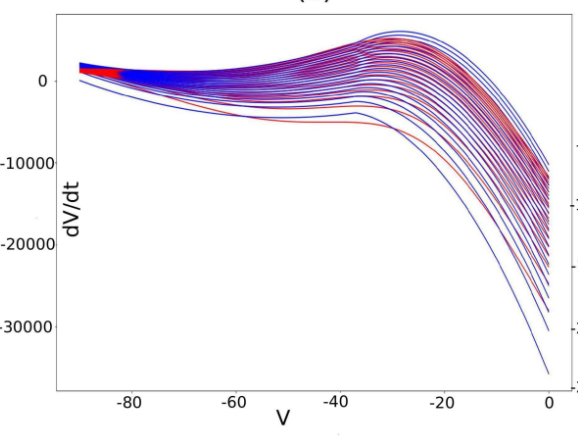

(e) $\left[\mathrm{K}^{+}\right]_{\text {bath }}=26.5$

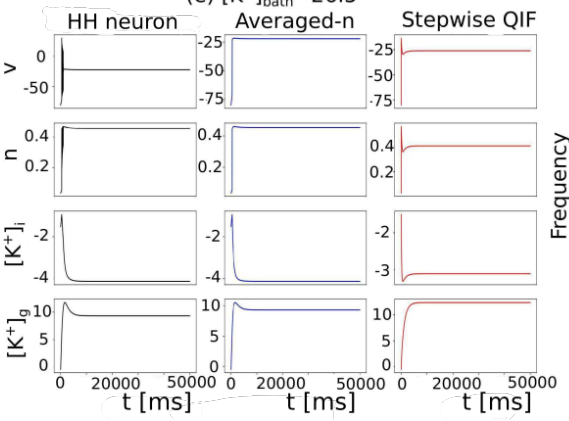

(c)

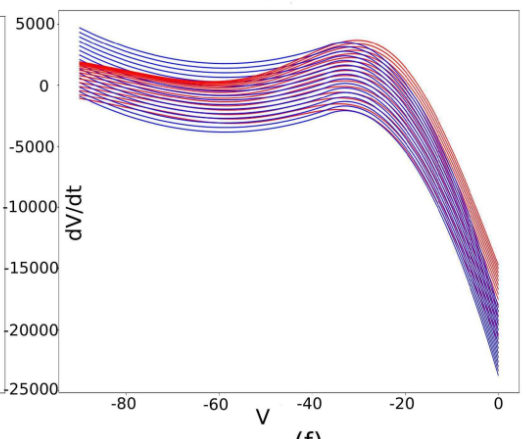

(f)

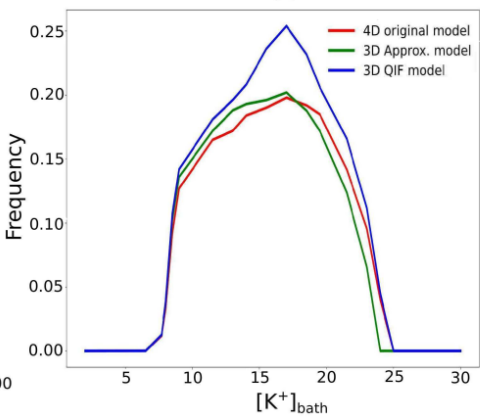

Figure 2. Dependence of $\frac{d V}{d t}[\mathrm{mV}]$ on $n$ : (a) $\frac{d V}{d t}[\mathrm{mV}]$ for different values of $n$ in cyan and the average trajectory in red. (b) Approximation of $\frac{d V}{d t}[\mathrm{mV}]$ for different values of $\Delta\left[K^{+}\right]_{i}\left[\mathrm{~mol} \backslash \mathrm{m}^{3}\right]$ : original functions in red and approximations in blue. (c) Approximation of $\frac{d V}{d t}[\mathrm{mV}]$ for different values of $\left[\mathrm{K}^{+}\right]_{\text {bath }}\left[\mathrm{mol} \backslash \mathrm{m}^{3}\right]$ : original functions in red and approximations in blue. (d-e) Simulation of the dynamics: time series of $V$ [mV] (in red), $n$ (in blue), $\Delta\left[K^{+}\right]_{i}\left[\mathrm{~mol} \backslash \mathrm{m}^{3}\right]$ (in green), and $[K]_{g}\left[\mathrm{~mol} \backslash \mathrm{m}^{3}\right]$ (in black): from the left original 4D system (1st column), 3D approximation (2nd column), 3D stepwise QIF approximation (3rd column); for (d) $\left[K^{+}\right]_{b a t h}=6.5$, (e) $\left[K^{+}\right]_{b a t h}=26.5$. (f) Frequency of oscillation: for the original 4D model in red; for the reduced 3D model in green; for the stepwise QIF approximation in blue.

where $f_{V}(t, V)$ represents the derivative of $f$ with respect to $V$. Integrating from last two ratios we got an independent solution in which $\rho$ is proportional to $\frac{1}{f(t, V)}$, i.e., $\rho \propto \frac{1}{f(t, V)}$. Hence, the trivial solution of the continuity equation Eq. (15) has the functional form $\rho_{0}\left(V, \Delta\left[K^{+}\right]_{i},\left[K^{+}\right]_{b a t h}, \eta, t\right) \propto\left(\frac{d V}{d t}\right)^{-1}$ that is inversely proportional to their time derivative for each value of $\eta$.

$$
\rho\left(V, \Delta\left[K^{+}\right]_{i},\left[K^{+}\right]_{b a t h}, \eta, t\right)= \begin{cases}\frac{K_{1}}{b_{1}\left\{\left(V-d_{1}\right)^{2}+I_{1}\right\}} ; & \forall V \geq s \\ \frac{K_{2}}{b_{2}\left\{\left(V-d_{2}\right)^{2}+I_{2}\right\}} ; & \forall V<s\end{cases}
$$

Here $k_{1}$ and $k_{2}$ are constants, so that the integral becomes one. Since $I_{1}, I_{2}, d_{1}$ and $d_{2}$ are functions of $\left[K^{+}\right]_{b a t h}$ and $\Delta\left[K^{+}\right]_{i}$ within the thermodynamic limit $N \rightarrow \infty$, the differential equation for the membrane potential can be represented as

$$
\frac{d V}{d t}=\left\{\begin{array}{l}
b_{1}\left\{\left[V-y\left(\eta, \Delta\left[K^{+}\right]_{i},\left[K^{+}\right]_{\text {bath }}, t\right)\right]^{2}+\left[x\left(\eta, \Delta\left[K^{+}\right]_{i},\left[K^{+}\right]_{\text {bath }}, t\right)\right]^{2}\right\} ; \forall V \geq s \\
b_{2}\left\{\left[V-y\left(\eta, \Delta\left[K^{+}\right]_{i},\left[K^{+}\right]_{\text {bath }}, t\right)\right]^{2}+\left[x\left(\eta, \Delta\left[K^{+}\right]_{i},\left[K^{+}\right]_{\text {bath }}, t\right)\right]^{2}\right\} ; \forall V<s
\end{array}\right.
$$

And the probability density function $\rho$ can be written as

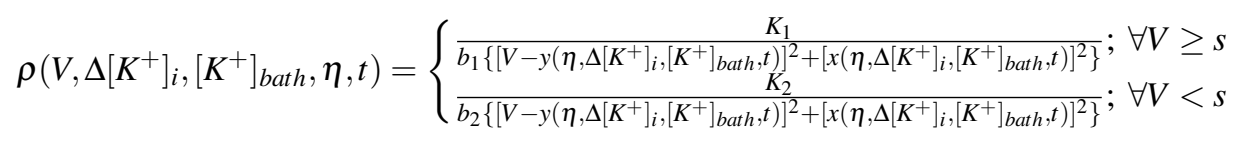

Since for each values of $\eta, \int_{-\infty}^{\infty} \rho d V=1$, we found solutions for constants $k_{1}=\frac{b_{1} x}{\pi}$ and $k_{2}=\frac{b_{2} x}{\pi}$ which reduces the relevant dynamics to a lower dimensional space and the solution of Eq. (15) converges to some Lorentzian type function independently of the initial condition. Then, the corresponding conditional probability can be expressed as a Lorentzian ansatz (LA) (Montbrió et al., 2015, Montbrió and Pazó, 2020), as follows:

$$
\rho\left(V, \Delta\left[K^{+}\right]_{i},\left[K^{+}\right]_{b a t h}, \eta, t\right)=\frac{1}{\pi} \frac{x\left(\eta, \Delta\left[K^{+}\right]_{i},\left[K^{+}\right]_{b a t h}, t\right)}{\left[V-y\left(\eta, \Delta\left[K^{+}\right]_{i},\left[K^{+}\right]_{b a t h}, t\right)\right]^{2}+\left[x\left(\eta, \Delta\left[K^{+}\right]_{i},\left[K^{+}\right]_{b a t h}, t\right)\right]^{2}}
$$


bioRxiv preprint doi: https://doi.org/10.1101/2021.10.29.466427; this version posted December 7, 2021. The copyright holder for this preprint (which was not certified by peer review) is the author/funder, who has granted bioRxiv a license to display the preprint in perpetuity. It is made

Here, $y\left(\eta, \Delta\left[K^{+}\right]_{i},\left[K^{+}\right]_{b a t h}, t\right)$ is related to the mean membrane potential of each value of $\eta$.

$$
\begin{aligned}
& \left\langle V\left(\eta, \Delta\left[K^{+}\right]_{i},\left[K^{+}\right]_{\text {bath }}, t\right)\right\rangle=\int_{-\infty}^{\infty} \rho\left(V, \eta, \Delta\left[K^{+}\right]_{i},\left[K^{+}\right]_{\text {bath }}, t\right) V d V=v\left(\eta, \Delta\left[K^{+}\right]_{i},\left[K^{+}\right]_{\text {bath }}, t\right)(\text { say }) \\
& =\int_{-\infty}^{\infty} \frac{1}{\pi} \frac{x\left(\eta, \Delta\left[K^{+}\right]_{i},\left[K^{+}\right]_{\text {bath }}, t\right)}{\left[V-y\left(\eta, \Delta\left[K^{+}\right]_{i},\left[K^{+}\right]_{\text {bath }}, t\right)\right]^{2}+\left[x\left(\eta, \Delta\left[K^{+}\right]_{i},\left[K^{+}\right]_{\text {bath }}, t\right)\right]^{2}} V d V \\
& =\frac{x}{\pi} \int_{-\infty}^{\infty} \frac{V-y+y}{(V-y)^{2}+x^{2}} d V=\frac{x}{\pi} \int_{-\infty}^{\infty}\left[\frac{V-y}{(V-y)^{2}+x^{2}} d V+\frac{y}{(V-y)^{2}+x^{2}} d V\right] \\
& =\frac{x}{\pi} \lim _{R \rightarrow \infty}\left[\frac{1}{2} \log \left(\frac{[R-y]^{2}+x^{2}}{[-R-y]^{2}+x^{2}}\right)+\frac{y}{x}\left[\tan ^{-1}(R)-\tan ^{-1}(-R)\right]\right]
\end{aligned}
$$

Taking the principal value of the Cauchy integral we can write the following:

$$
v\left(\eta, \Delta\left[K^{+}\right]_{i},\left[K^{+}\right]_{b a t h}, t\right)=y\left(\eta, \Delta\left[K^{+}\right]_{i},\left[K^{+}\right]_{b a t h}, t\right) ; \text { and } \quad v(t)=\int_{-\infty}^{\infty} y\left(\eta, \Delta\left[K^{+}\right]_{i},\left[K^{+}\right]_{b a t h}, t\right) g(\eta) d \eta
$$

Now, we solved the continuity equation Eq. (15) with LA (Eq. (20))

$$
\delta_{t} \rho=\frac{1}{\pi} \frac{\left[(V-y)^{2}+x^{2}\right] \dot{x}-x[-2(V-y)+2 x \dot{x}]}{\left[(V-y)^{2}+x^{2}\right]^{2}}=\frac{1}{\pi} \frac{\dot{x} V^{2}-2(y \dot{x}+x \dot{y}) V+\left(\left[y^{2}-x^{2}\right] \dot{x}-2 x y \dot{y}\right)}{\left[(V-y)^{2}+x^{2}\right]^{2}}
$$

Here overdot represents the derivative with respect to time. We took the rate of change of the membrane potential as Eq. (13) and obtained

$$
\begin{gathered}
\delta_{V}(\dot{V} \rho)=\ddot{V} \rho+\dot{V} \delta_{V}(\rho)=\left\{\begin{array}{l}
\frac{1}{\pi} \frac{\left(2 b_{1}\left(V-d_{1}\right)+J g_{s y n}\right) x}{\left[(V-y)^{2}+x^{2}\right]}-\frac{2 x \dot{V}(V-y)}{\pi\left[(V-y)^{2}+x^{2}\right]^{2}} ; \forall V \geq s \\
\frac{1}{\pi} \frac{\left(2 b_{2}\left(V-d_{2}\right)+J g_{s y n}\right) x}{\left[(V-y)^{2}+x^{2}\right]}-\frac{2 x \dot{V}(V-y)}{\pi\left[(V-y)^{2}+x^{2}\right]^{2}} ; \forall V<s
\end{array}=\right. \\
\left\{\begin{array}{l}
\frac{\left[2 b_{1} d_{1} x-2 b_{1} x y-J g_{s y n} x\right] V^{2}+2\left[b_{1} x^{3}+b_{1} x y^{2}-b_{1} d_{1}^{2} x-b_{1} I_{1} x+J g_{s y n} E x\right] V+\left[\left(J g_{s y n}-2 b_{1} d_{1}\right)\left(x^{2}+y^{2}\right) x+2\left(b_{1} d_{1}^{2}+b_{1} I_{1}-J g_{s y n} E\right) x y+\left(y^{2}-x^{2}\right) \dot{x}-2 x y \dot{y}\right]}{\pi\left[(V-y)^{2}+x^{2}\right]^{2}} ; \forall V \geq s \\
\frac{\left[2 b_{2} d_{2} x-2 b_{2} x y-J g_{s y n} x\right] V^{2}+2\left[b_{2} x^{3}+b_{2} x y^{2}-b_{2} d_{2}^{2} x-b_{2} I_{2} x+J g_{s y n} E x\right] V+\left[\left(J g_{s y n}-2 b_{2} d_{2}\right)\left(x^{2}+y^{2}\right) x+2\left(b_{2} d_{2}^{2}+b_{2} I_{2}-J g_{s y n} E\right) x y+\left(y^{2}-x^{2}\right) \dot{x}-2 x y \dot{y}\right]}{\pi\left[(V-y)^{2}+x^{2}\right]^{2}} ; \forall V<s
\end{array}\right.
\end{gathered}
$$

Hence, equating continuity equation Eq. (15) for being an identity, that is only if all the coefficients of the powers of $V$ are zero, we get (from the coefficient of $V^{2}=0$ )

$$
\dot{x}=\left\{\begin{array}{l}
{\left[2 b_{1}\left(y-d_{1}\right)+J g_{s y n}\right] x ; \forall V \geq s} \\
{\left[2 b_{2}\left(y-d_{2}\right)+J g_{s y n}\right] x ; \forall V<s}
\end{array}\right.
$$

From the coefficient of $V=0$ we get

$$
\begin{aligned}
& \left\{\begin{array}{l}
b_{1} x^{3}+b_{1} x y^{2}-b_{1} d_{1}^{2} x-b_{1} I_{1} x+J g_{s y n} E x-y \dot{x}+x \dot{y}=0 ; \forall V \geq s \\
b_{2} x^{3}+b_{2} x y^{2}-b_{2} d_{2}^{2} x-b_{2} I_{2} x+J g_{s y n} E x-y \dot{x}+x \dot{y}=0 ; \forall V<s
\end{array}\right. \\
& \Rightarrow \dot{y}=\left\{\begin{array}{l}
b_{1}\left[\left(y-d_{1}\right)^{2}+I_{1}-x^{2}\right]+J g_{s y n}(y-E) ; \forall V \geq s \\
b_{2}\left[\left(y-d_{2}\right)^{2}+I_{2}-x^{2}\right]+J g_{s y n}(y-E) ; \forall V<s
\end{array}\right.
\end{aligned}
$$

This leads to the constant term to be zero and we obtained the mean-field model, as follows:

$$
\begin{aligned}
\dot{x} & =\left\{\begin{array}{l}
{\left[2 b_{1}\left(y-d_{1}\right)+J g_{s y n}\right] x ; \forall V \geq s} \\
{\left[2 b_{2}\left(y-d_{2}\right)+J g_{s y n}\right] x ; \forall V<s}
\end{array}\right. \\
\dot{y} & =\left\{\begin{array}{l}
b_{1}\left[\left(y-d_{1}\right)^{2}+I_{1}-x^{2}\right]+J g_{s y n}(y-E) ; \forall V \geq s \\
\dot{y}=b_{2}\left[\left(y-d_{2}\right)^{2}+I_{2}-x^{2}\right]+J g_{s y n}(y-E) ; \forall V<s
\end{array}\right.
\end{aligned}
$$

This pair of equations can be represented by a single complex valued equation, as follows:

$$
\delta_{t} \omega\left(\eta, \Delta\left[K^{+}\right]_{i},\left[K^{+}\right]_{b a t h}, t\right)=\left\{\begin{array}{l}
i b_{1}\left[\left(i \omega+d_{1}\right)^{2}+I_{1}\right]+J g_{s y n}[i \omega+2 y-E] ; \forall V \geq s \\
i b_{2}\left[\left(i \omega+d_{2}\right)^{2}+I_{2}\right]+J g_{s y n}[i \omega+2 y-E] ; \forall V<s
\end{array}\right.
$$


where $\omega\left(\eta, \Delta\left[K^{+}\right]_{i},\left[K^{+}\right]_{b a t h}, t\right)=x\left(\eta, \Delta\left[K^{+}\right]_{i},\left[K^{+}\right]_{b a t h}, t\right)+i y\left(\eta, \Delta\left[K^{+}\right]_{i},\left[K^{+}\right]_{b a t h}, t\right)$ and real and complex components of Eq. (25) represent the dynamics of $y$ and $x$ respectively. Moreover, if we consider the distribution of the heterogeneous input current $\eta$ to be a Lorentzian distribution with half-width $\Delta$ and location of the center be $\bar{\eta}$, i.e. :

$$
g(\eta)=\frac{1}{\pi} \frac{\Delta}{(\eta-\bar{\eta})^{2}-\Delta^{2}}
$$

Then, the residue theorem can be applied to compute the integral in Eq. (21) over the closed contour in the complex $\eta$-plane. It should be noted that the assumption of $g(\eta)$ as a Lorentzian distribution leads to the conclusion that $v(t)$ and $x(t)$ could be computed by Cauchy residue theorem with the value of $\omega$ at $\eta=\bar{\eta}-i \Delta$, i.e., at the pole of the Lorentzian distribution Eq. (26) in the lower half of $\eta$-plane: $x\left(\Delta\left[K^{+}\right]_{i},\left[K^{+}\right]_{b a t h}, t\right)+i y\left(\Delta\left[K^{+}\right]_{i},\left[K^{+}\right]_{b a t h}, t\right)=\omega\left(\bar{\eta}-i \Delta, \Delta\left[K^{+}\right]_{i},\left[K^{+}\right]_{b a t h}, t\right)$. Thus evaluating Eq. (26) at $\eta=\bar{\eta}-i \Delta$ we obtained the mean-field model of membrane potential in terms of two coupled differential equations considering non-autonomous slow variables, as follows:

$$
\begin{aligned}
& \dot{x}=\left\{\begin{array}{l}
b_{1}\left(\Delta+2 x\left(v-d_{1}\right)\right)+J g_{s y n} x ; \forall v \geq s \\
b_{2}\left(\Delta+2 x\left(v-d_{2}\right)\right)+J g_{s y n} x ; \forall v<s
\end{array}\right. \\
& \dot{v}=\left\{\begin{array}{l}
b_{1}\left[\left(v-d_{1}\right)^{2}+\bar{\eta}+I_{1}-x^{2}\right]+J g_{s y n}(v-E) ; \forall v \geq s \\
b_{2}\left[\left(v-d_{2}\right)^{2}+\bar{\eta}+I_{2}-x^{2}\right]+J g_{s y n}(v-E) ; \forall v<s
\end{array}\right.
\end{aligned}
$$

Hence, the 4-dimensional mean-field equation considering the dynamics for the slow variables $\Delta\left[K^{+}\right]_{i}$ and $\left[K^{+}\right]_{g}$ becomes the following:

$$
\begin{aligned}
\frac{d x}{d t} & =\left\{\begin{array}{l}
{\left[b_{1}\left(\Delta+2 x\left(v-d_{1}\right)\right)+J g_{s y n} x ; \forall V \geq s\right.} \\
{\left[b_{2}\left(\Delta+2 x\left(v-d_{2}\right)\right)+J g_{s y n} x ; \forall V<s\right.}
\end{array}\right. \\
\frac{d v}{d t} & =\left\{\begin{array}{l}
b_{1}\left[\left(v-d_{1}\right)^{2}+\bar{\eta}+I_{1}-x^{2}\right]+J g_{s y n}(v-E) ; \forall V \geq s \\
b_{2}\left[\left(v-d_{2}\right)^{2}+\bar{\eta}+I_{2}-x^{2}\right]+J g_{s y n}(v-E) ; \forall V<s
\end{array}\right. \\
\frac{d \Delta\left[K^{+}\right]_{i}}{d t} & =-\frac{\gamma}{w_{i}}\left(I_{k}(v)-2 I_{\text {pump }}\right) \\
\frac{d\left[K^{+}\right]_{g}}{d t} & =\varepsilon\left(\left[K^{+}\right]_{\text {bath }}+\beta \Delta\left[K^{+}\right]_{i}-\left\{\left[K^{+}\right]_{0, o}+\left[K^{+}\right]_{g}\right\}\right)
\end{aligned}
$$

Here $v$ denotes the mean membrane potential, $x$ is a phenomenological variable, and intracellular potassium concentration variation and extracellular potassium buffering by the external bath are denoted by $\Delta\left[K^{+}\right]_{i}$ and $[K]_{g}$ respectively. It should be noted that $x$ and its dynamics only depend upon $v$ by construction, so technically, the dynamical system in Eq. (27) could be characterized by only one fast variable $v$. Consequently, with only one fast variable the dynamics cannot demonstrate fast oscillations but only the envelope of the oscillations, as demonstrated in Fig. 3. Therefore, the spike train and tonic spike features in the corresponding regime of the dynamics, which are characterized by sharp oscillations in the $v$ and $n$ dimensions alone, are lost through averaging out $n$ from the system Eq. (1).

Moreover, the variable $n$ represents the probability of channel opening, and in principle, the expected probability of channel opening is proportional to the synaptic conductance $g_{s y n}$ (i.e. $g_{s y n} \propto n$ ). Therefore, at this point we defined the normalized synaptic conductance $g_{s y n}$ as $c n$ ( $c$ being the normalized constant of proportionality) within the thermodynamic limit. This also allowed us to replace the step-wise QIF approximation by the original functional forms in the mean-field model, as Eq. (13) is an approximation of Eq. (12) in the first place. Here we can replace the step-wise quadratic functions in Eq. (13) by the original function from Eq. (12), including the synaptic conductance variable $n$. Hence, the final mean-field approximation is a 
bioRxiv preprint doi: https://doi.org/10.1101/2021.10.29.466427; this version posted December 7, 2021. The copyright holder for this preprint (which was not certified by peer review) is the author/funder, who has granted bioRxiv a license to display the preprint in perpetuity. It is made 10 available under aCC-BY-NC-ND 4.0 International license.

Biophysical neural mass model of ion-exchange

(a)
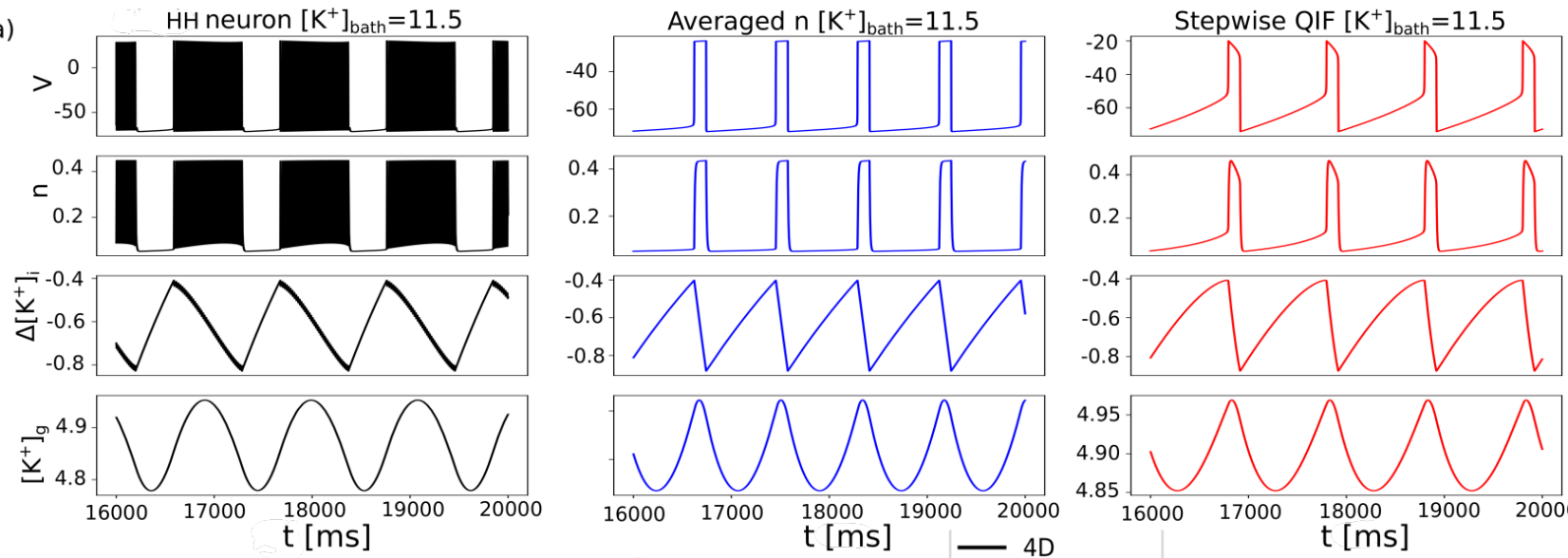

(b)

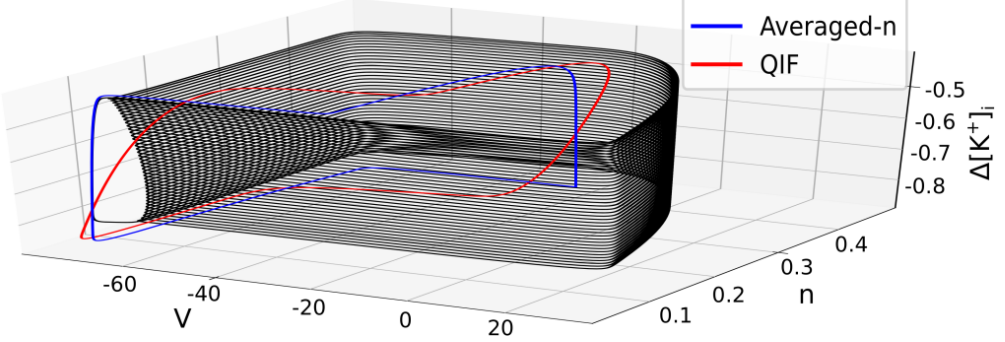

Figure 3. Simulation of the dynamics: (a) Time series of $V[\mathrm{mV}]$ (1st row), $n$ (2nd row), $\Delta\left[K^{+}\right]_{i}\left[\mathrm{~mol} \backslash \mathrm{m}^{3}\right]$ (3rd row), and $[K]_{g}$ $\left[\mathrm{mol} \backslash \mathrm{m}^{3}\right]$ (4th row): from the left original 4D system (in black), 3D approximation (in blue), 3D stepwise QIF approximation (in red); (b) 3D Phase space with $V[\mathrm{mV}], n$, and $\Delta\left[K^{+}\right]_{i}\left[\mathrm{~mol} \backslash \mathrm{m}^{3}\right]$ for three models with the original 4D model in black, for the reduced 3D model in blue, and for the stepwise QIF approximation in red; for $\left[K^{+}\right]_{\text {bath }}=11.5$.

five-dimensional dynamical system as follows:

$$
\begin{aligned}
\frac{d x}{d t} & =\left\{\begin{array}{l}
b_{1}\left(\Delta+2 x\left(v-d_{1}\right)\right)+c J n x ; \forall V \geq s \\
b_{2}\left(\Delta+2 x\left(v-d_{2}\right)\right)+c J n x ; \forall V<s
\end{array}\right. \\
\frac{d v}{d t} & =\left\{\begin{array}{l}
-\frac{1}{C_{m}}\left(J_{C l}+J_{N a}+J_{K}+J_{\text {pump }}\right)-b_{1} x^{2}+\bar{\eta}+c J n(v-E) ; \forall V \geq s \\
-\frac{1}{C_{m}}\left(J_{C l}+J_{N a}+J_{K}+J_{\text {pump }}\right)-b_{2} x^{2}+\bar{\eta}+c J n(v-E) ; \forall V<s
\end{array}\right. \\
\frac{d n}{d t} & =\frac{1}{\tau_{n}}\left(\frac{1}{1+\exp \frac{-19-V}{18}}-n\right) \\
\frac{d \Delta\left[K^{+}\right]_{i}}{d t} & =-\frac{\gamma}{w_{i}}\left(I_{k}(v)-2 I_{\text {pump }}\right) \\
\frac{d\left[K^{+}\right]_{g}}{d t} & =\varepsilon\left(\left[K^{+}\right]_{\text {bath }}+\beta \Delta\left[K^{+}\right]_{i}-\left\{\left[K^{+}\right]_{0, o}+\left[K^{+}\right]_{g}\right\}\right)
\end{aligned}
$$

If we consider the inhibitory and excitatory group of neurons, the corresponding mean-field approximation can be written as 
follows:

$$
\begin{aligned}
\frac{d x}{d t} & =\left\{\begin{array}{l}
b_{1}\left(\Delta+2 x\left(v-d_{1}\right)\right)+\left(c_{i}+c_{e}\right) J n x ; \forall V \geq s \\
b_{2}\left(\Delta+2 x\left(v-d_{2}\right)\right)+\left(c_{i}+c_{e}\right) J n x ; \forall V<s
\end{array}\right. \\
\frac{d v}{d t} & =\left\{\begin{array}{l}
-\frac{1}{C_{m}}\left(J_{C l}+J_{N a}+J_{K}+J_{\text {pump }}\right)-b_{1} x^{2}+\bar{\eta}+c_{i} J n\left(v-E_{i}\right)+c_{e} J n\left(v-E_{e}\right) ; \forall V \geq s \\
-\frac{1}{C_{m}}\left(J_{C l}+J_{N a}+J_{K}+J_{\text {pump }}\right)-b_{2} x^{2}+\bar{\eta}+c_{i} J n\left(v-E_{i}\right)+c_{e} J n\left(v-E_{e}\right) ; \forall V<s
\end{array}\right. \\
\frac{d n}{d t} & =\frac{1}{\tau_{n}}\left(\frac{1}{\left.1+\exp \frac{-19-V}{18}-n\right)}\right. \\
\frac{d \Delta\left[K^{+}\right]_{i}}{d t} & =-\frac{\gamma}{w_{i}}\left(I_{k}(v)-2 I_{\text {pump }}\right) \\
\frac{d\left[K^{+}\right]_{g}}{d t} & =\varepsilon\left(\left[K^{+}\right]_{\text {bath }}+\beta \Delta\left[K^{+}\right]_{i}-\left\{\left[K^{+}\right]_{0, o}+\left[K^{+}\right]_{g}\right\}\right)
\end{aligned}
$$

Where $E_{e}$ and $E_{i}$ are the synaptic reversal potentials of the excitatory and inhibitory groups of neurons.

\section{Results}

In this paper we have derived a mean-field approximation of locally homogeneous network of Hodgkin-Huxley type neurons (HH neuron) with heterogeneous quenched external input $\eta$ (Eq. (28), and Eq. (29)). The appropriateness of mean-field approximation is validated by comparing the simulation results of the large network of coupled HH neurons with the mean-field model Eq. (29) which appears to be quite robust under stochasticity and different type of distribution of heterogeneous quenched external input $\eta$ (Fig. 4). Our model demonstrates different types of spiking and bursting behavior as well as resting-state (Fig. 6) and multistability in the distinct regime of external potassium bath $\left(\left[\mathrm{K}^{+}\right]_{\text {bath }}\right)$. These kinds of neuronal activities are quite observable in large-scale brain dynamics which by the means of this novel mean-field model get connected with the ion-exchange mechanism and the ion concentration states in the cellular space. The bifurcation analysis of the mean-field model is carried out to identify relevant parameter regime which is responsible for different types of spiking and bursting behavior, resting-state, and multistability features in the mean-field model (Fig. 5). Moreover, it is observed that even if in the resting state (healthy) network certain kinds of external stimulus current could generate transient neuronal bursts (in terms of transition between upstate and downstate) which are validated by parallel simulation of the large network of coupled HH neurons and the mean-field model (Fig. 7). This result could be quite significant in terms of explaining the neuronal activities during brain stimulation. Furthermore, the behavior of two of such mean-field representations under the aforementioned conductance-based coupling (Eq. (9)) are demonstrated (Fig. 8) and validated with the simulation of two groups of neuron with different parameter. It could serve as a baseline to explain how epileptic seizures could propagate through some healthy brain regions as a result of their coupling with epileptogenic regions.

The simulation of Hodgkin-Huxley type single neuron dynamics Eq. (1) driven by an ion-exchange mechanism, has revealed that the parameter $\left[\mathrm{K}^{+}\right]_{\text {bath }}$ is the most important parameter for describing the dynamics. Previous studies showed that changing the value of the concentration of the external potassium bath is responsible for qualitative changes in the dynamical behavior of a single neuronal system (Depannemaecker et al., 2021), particularly discovering that in the parameter regime $\left[K^{+}\right]_{\text {bath }}=[6.01,6.875]$ multistability occurs.

Reproducing the bifurcation analysis by a numerical continuation revealed two stable fixed points and a stable focus in this regime for $\left[K^{+}\right]_{\text {bath }}$. We also observed a Hopf bifurcation at $\left[K^{+}\right]_{\text {bath }}=7.68$. At higher values of $\left[K^{+}\right]_{\text {bath }}$ he stable fixed-point behavior of the dynamics changes into limit cycle behavior and the dynamics shows the bursting characteristics of neurons. The single neuron model was also shown to be able to produce different dynamic behaviors of single neuronal activity, such as epileptic bursts, status epilepticus, resting state, etc. In this study we developed a mean-field dynamics system based on a biophysical single neuron model Eq. (1) to describe the network behavior of biophysical neurons within the thermodynamic limit.

To be able to apply convenient mathematical (analytical) methods in this study, the velocity of the membrane potential of a single neuron described by Eq. (1) was approximated as a stepwise quadratic function because geometrically it resembles a combination of two inverted parabolas. The validity of this approximation is shown in Fig. 2 where the time derivative of $V$ in Eq. (1) is compared with that of a stepwise quadratic approximation. The appropriateness of the approximation of the membrane potential dynamics is represented for different values of the other state variables and parameters, such as $n, \Delta\left[K^{+}\right]_{i}$, and $\left[K^{+}\right]_{\text {bath }}$ in Fig. 2(a), 2(b), and 2(c), respectively. 
bioRxiv preprint doi: https://doi.org/10.1101/2021.10.29.466427; this version posted December 7, 2021. The copyright holder for this preprint (which was not certified by peer review) is the author/funder, who has granted bioRxiv a license to display the preprint in perpetuity. It is made 12 available under aCC-BY-NC-ND 4.0 International license.

Biophysical neural mass model of ion-exchange

(a) Non-autonomous slow variables

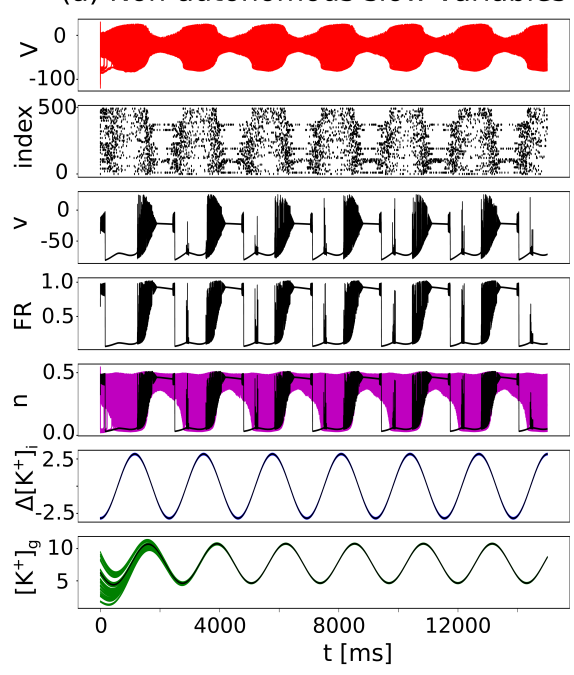

(d) Gaussian heterogeneity
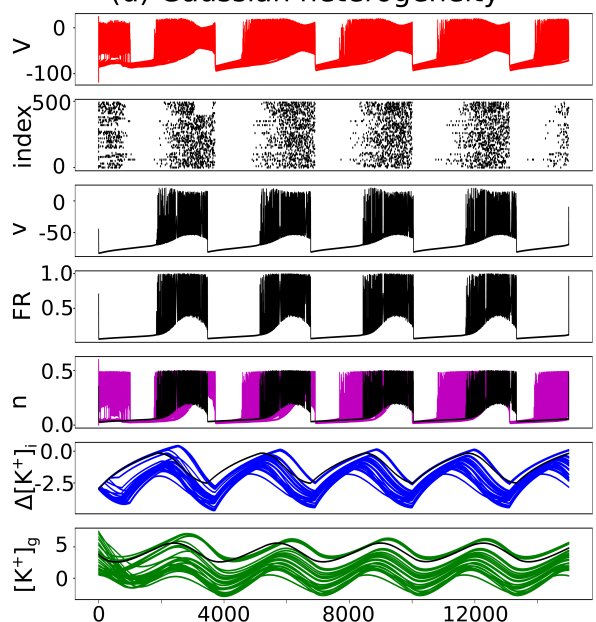

$\mathrm{t}[\mathrm{ms}]$ (b)

(b1) Network of 4D HH neurons

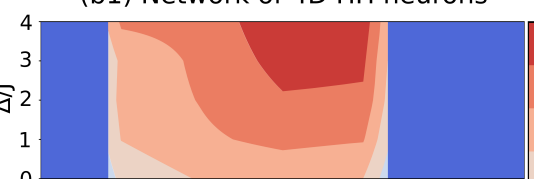

(b2) 5D mean field model

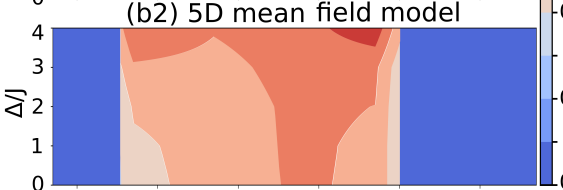

(b3) Difference (b1) - (b2)

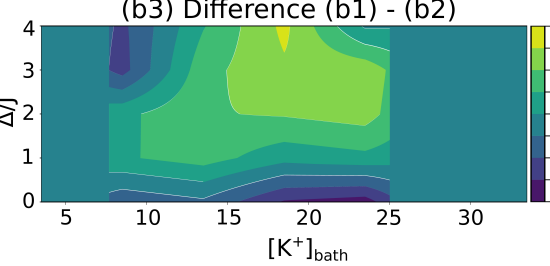

(e) Stochastic noise (low heterogeneity) (c) Lorentzian heterogeneity

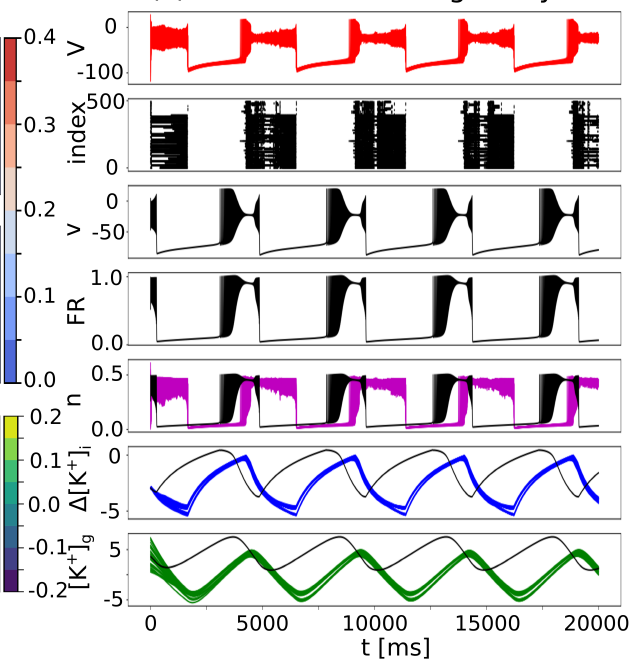

(f) Stochastic noise (high heterogeneity)

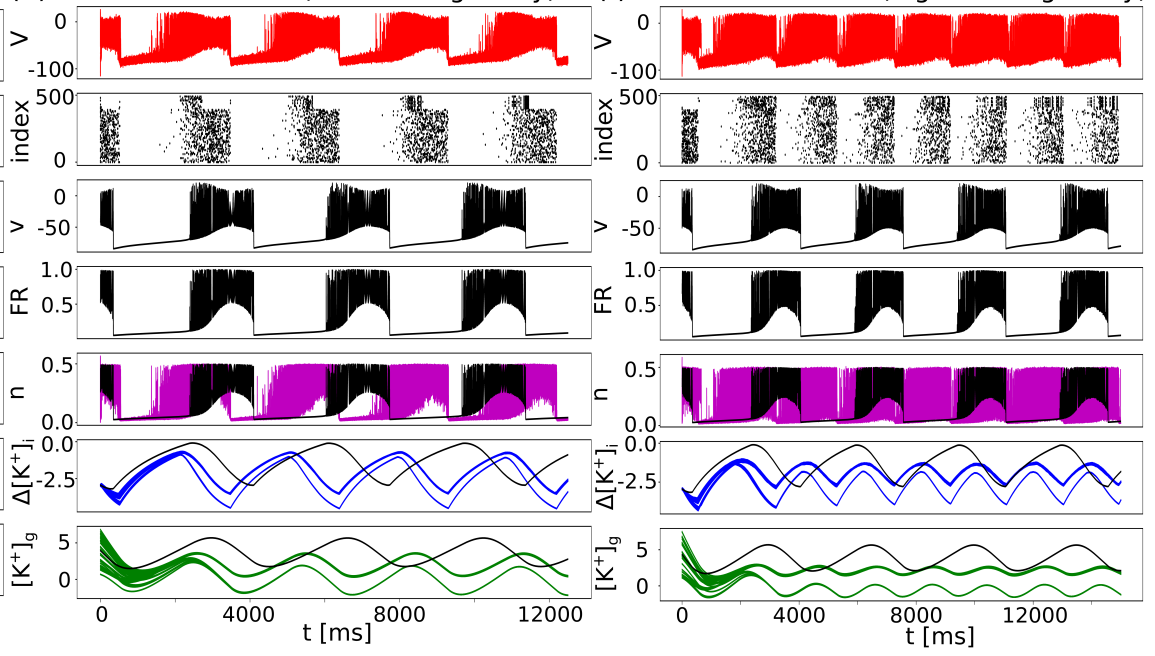

Figure 4. Simulations of networks of neurons with the mean-field model: $V[\mathrm{mV}]$ for the network nodes in the first row (in red); scatter of the firing neurons in the second row; $v[\mathrm{mV}]$ for the mean-field in the third row (in black); firing rate of the mean-field model in the fourth row; $n$ in the fifth row: network nodes in magenta and mean-field in black; $\Delta\left[K^{+}\right]_{i}\left[\mathrm{~mol}_{\backslash m^{3}}\right]$ in the sixth row: network nodes in blue and mean-field in black, and $\left[K^{+}\right]_{g}\left[\mathrm{~mol} \backslash \mathrm{m}^{3}\right]$ in the seventh row: network nodes in green and mean-field in black, for $\left[K^{+}\right]_{\text {bath }}=12.5, J=4$ : (a) Non-autonomous $\Delta\left[K^{+}\right]_{i}$ for $\frac{\Delta}{J}=3.0$, with $\Delta\left[K^{+}\right]_{i}$ as in Eq. (29); (c) Lorentzian distribution of heterogeneity $\eta$ for $\frac{\Delta}{J}=1.0$; (d) Gaussian distribution of heterogeneity $\eta$ for $\frac{\Delta}{J}=4.0$, with stochastic white noise $\eta$; (e) $\frac{\Delta}{J}=2.0$ and (f) $\frac{\Delta}{J}=3.0$. (b) Frequencies (represented by the color code) of the network of coupled $\mathrm{HH}$ neurons as a function of $\left[\mathrm{K}^{+}\right]_{\text {bath }}$ and relative half-width of heterogeneity $\frac{\Delta}{J}$ in the first row (b1), frequencies (represented by the color code) of the mean-field model as a function of $\left[K^{+}\right]_{\text {bath }}$ and relative half-width of heterogeneity $\frac{\Delta}{J}$ in the second row (b2), difference between frequencies (represented by the color code) from the network of coupled HH neurons and 5D-mean-field model in the third row: for (b3) $J=4$.

\section{Step-wise quadratic approximation for the nullcline}

The approximation of the membrane potential dynamics as a stepwise quadratic function allowed us to apply analytical approaches and to solve the corresponding continuity equation to derive the mean-field of a locally homogeneous mesoscopic network of biophysical neurons. Fig. 2(d, e) and Fig. 3(a) demonstrate the validity of the stepwise QIF approximation of the original dynamics for different values of $\left[K^{+}\right]_{\text {bath }}$ in terms of the simulation of the corresponding dynamics. These figures show that approximating the stepwise QIF captures the behavior of the original 4-dimensional dynamics for the entire biophysical range of $\left[K^{+}\right]_{\text {bath }}$. Fig. 3(b) illustrates the method for averaging the variable in a 3-dimensional phase space 
bioRxiv preprint doi: https://doi.org/10.1101/2021.10.29.466427; this version posted December 7, 2021. The copyright holder for this preprint (which was not certified by peer review) is the author/funder, who has granted bioRxiv a license to display the preprint in perpetuity. It is made Biophysical neural mass model of ion-exchange available under aCC-BY-NC-ND 4.0 International license.

(a) 3D bifurcation diagram

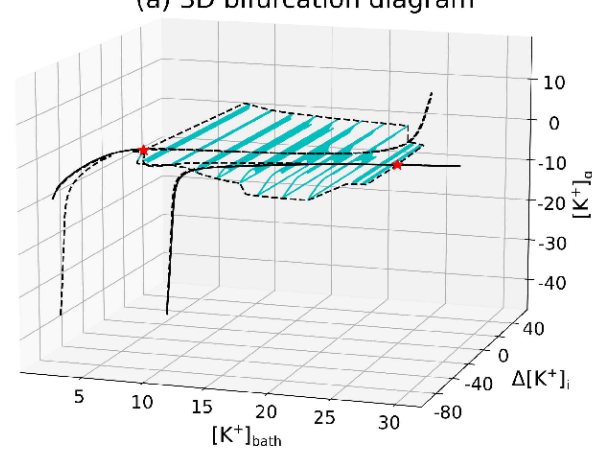

(b) $2 \mathrm{D}$ bifurcation diagram with respect to $\left[\mathrm{K}^{+}\right]_{\text {ba }}$

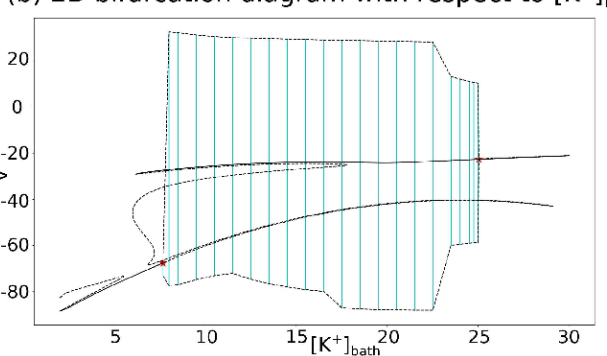

(c) Healthy state

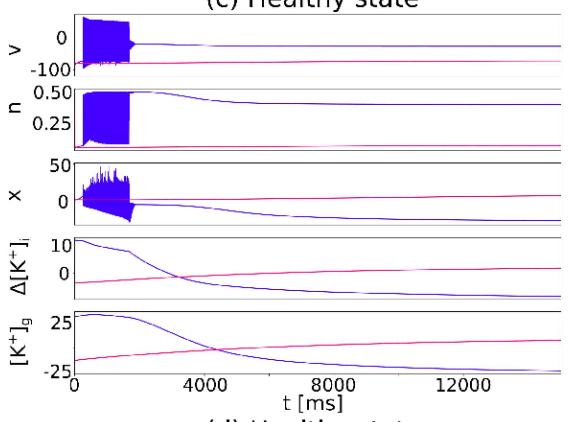

(d) Healthy state

Figure 5. Bifurcation diagram of the mean-field model: (a) $v[\mathrm{mV}]$ and $\Delta\left[K^{+}\right]_{i}\left[\mathrm{~mol} \backslash \mathrm{m}^{3}\right]$ are represented against $\left[K^{+}\right]_{\text {bath }}$ $\left[\mathrm{mol} \backslash \mathrm{m}^{3}\right]$, (b) $v[\mathrm{mV}]$ is represented against $\left[K^{+}\right]_{\text {bath }}\left[\mathrm{mol} \backslash \mathrm{m}^{3}\right]$, the stable fixed points are shown by black lines whereas the unstable fixed points are represented by dotted lines. The maximum and minimum values of the limit cycle are shown by black dotted lines in between the two bifurcation values are shown by red marks. Multistability in the mean-field model at $\left[K^{+}\right]_{\text {bath }}=7.65$ (healthy regime): (c) time series for healthy state $\left(\left[K^{+}\right]_{b a t h}=7.65\right)$ demonstrating the up and down states, up state in blue and down state in red. (d) Three-dimensional phase space in $v, \Delta\left[K^{+}\right]_{i}$, and $\left[K^{+}\right]_{g}$ dimensions for the up and down states: the black marks denote the fixed points in the up and down state. Multistability in the mean-field model at $\left[K^{+}\right]_{\text {bath }}=11.5$ and 18.5 (bursting regime): (e) time series for the up and down states: epileptic state in violet, bursting in blue, up states in pink, and red for different values of $\left[K^{+}\right]_{b a t h}$. (f) Three-dimensional phase space in $v, \Delta\left[K^{+}\right]_{i}$, and $\left[K^{+}\right]_{g}$

dimensions for the up and down states (bursting): the black marks denote the fixed points up states, while the blue and violet phase space trajectories shows the bursting, and epileptic state.

portrait and demonstrate that averaging the variable $n$ Eq. (8) and the subsequent stepwise QIF approximation (Eq. (13) with no coupling or $J=0$ ) still preserve the slow dynamics is shown with 3-dimensional phase space portraits of each of the neurons for $\left[\mathrm{K}^{+}\right]_{\text {bath }}=11.5$. In Fig. 2(f) the frequencies of these three dynamics are compared for entire range of $\left[\mathrm{K}^{+}\right]_{\text {bath }}$ to demonstrate that, though there is a small frequency mismatch between these approximations, the overall stepwise QIF approximation captured the slow dynamic behavior of the original complex biophysical process described in Eq. (1) qualitatively as well as quantitatively. These allowed us to apply a Lorentzian distribution for the membrane potential and to develop the mean-field approximation of a heterogeneous network of biophysical neurons driven by ion-exchange dynamics coupled all-to-all via conductance-based coupling Eq. (11).

\section{Limits of the approximation}

When $\Delta\left[K^{+}\right]_{i}$ is non-autonomous, the mean-field model captures the exact frequency of the oscillation as it is analytically derived by substituting the LA into the continuity equation (12). This is demonstrated in Fig. 4(a) for a non-autonomous $\Delta\left[\mathrm{K}^{+}\right]_{i}$ taken as a sinusoidal function. When $\Delta\left[\mathrm{K}^{+}\right]_{i}$ is autonomous (function of state variables only), the mean-field model is a mean-field approximation, which encounters a frequency mismatch with the locally homogeneous mesoscopic network of biophysical neurons described in Eq. (1). Fig. 4(c) illustrates the validity of the mean-field approximation by a simulated plot from the network of biophysical neurons and the plot of the mean-field approximation described in Eq. (29). The frequencies of the mean-field model and the network of biophysical neurons again encounter a slight mismatch, which is illustrated in Fig. 4(b) for different values of relative heterogeneity $\Delta / J$. The frequency of oscillation of the network of neurons, the mean-field approximation, and their relative differences are represented in Fig. 4(b1), 4(b2), 4(b3), respectively, with respect to $\left[\mathrm{K}^{+}\right]_{\text {bath }}$ 
(a) Spike train, $\left[\mathrm{K}^{+}\right]_{\text {bath }}=8.5$

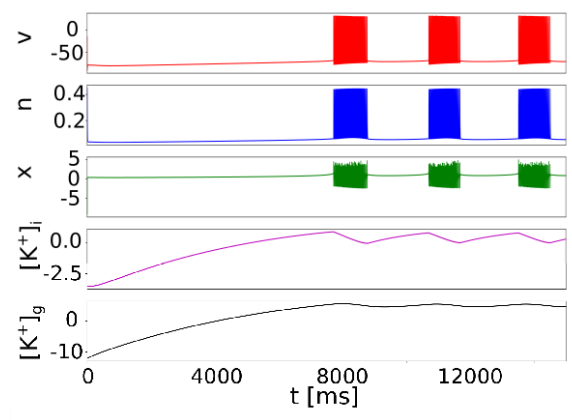

(d) Seizure-like event, $\left[\mathrm{K}^{+}\right]_{\text {bath }}=18.5$

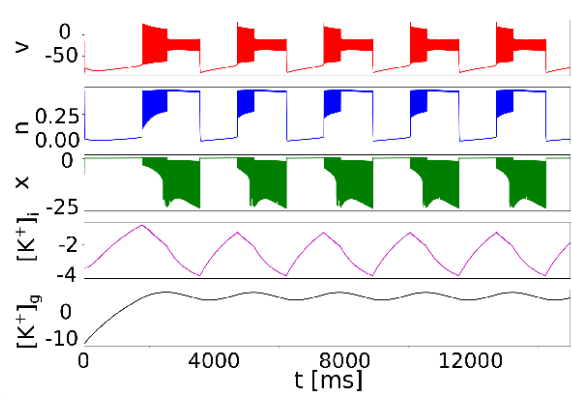

(b) Tonic spike, $\left[\mathrm{K}^{+}\right]_{\mathrm{bath}}=10.5$

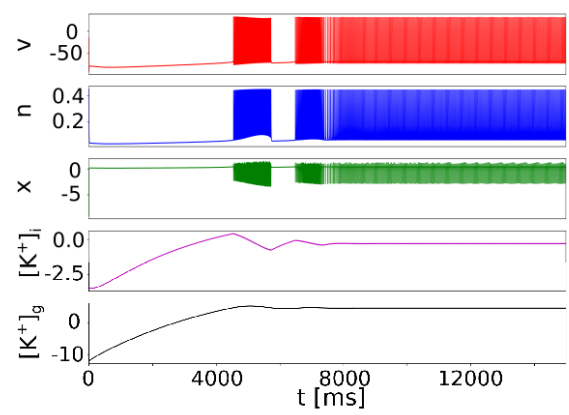

(e) Status epilepticus, $\left[\mathrm{K}^{+}\right]_{\text {bath }}=24.5$

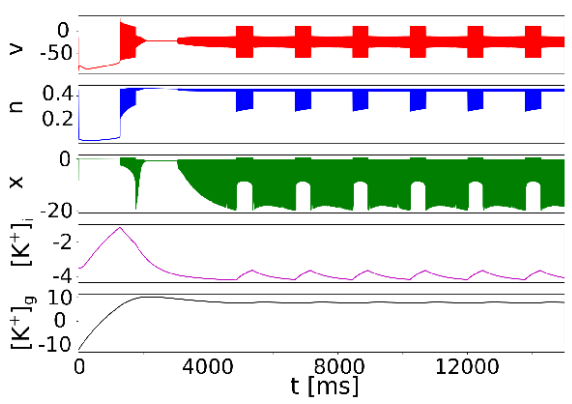

(c) Bursting, $\left[\mathrm{K}^{+}\right]_{\mathrm{bath}}=14.5$

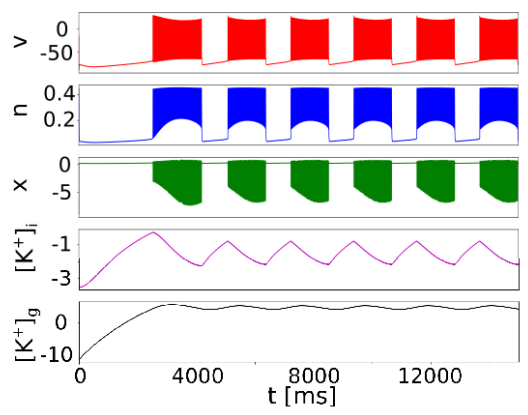

(f) Depolarization block, $\left[\mathrm{K}^{+}\right]_{\text {bath }}=25.5$

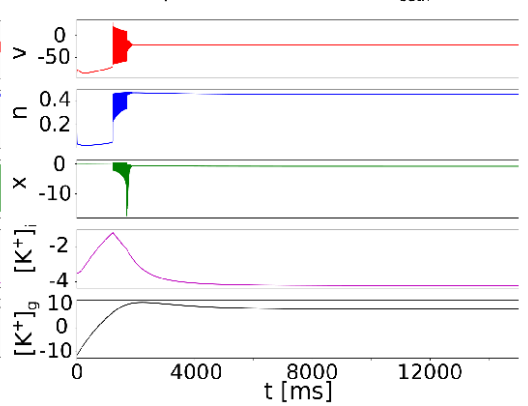

Figure 6. Qualitative mode of behavior of the mean-field model: time series $v[\mathrm{mV}]$ (in red), $n$ (in blue), $\Delta\left[K^{+}\right]_{i}\left[\mathrm{~mol} \backslash \mathrm{m}^{3}\right]$ (in green), and $\left[K^{+}\right]_{g}\left[\mathrm{~mol} \backslash \mathrm{m}^{3}\right]$ (in black) are demonstrated for the following patterns of activity: (a) Spike train at $\left[K^{+}\right]_{\text {bath }}=8.5$, (b) Tonic spiking (TS) at $\left[K^{+}\right]_{\text {bath }}=10.5$, (c) Bursting at $\left[K^{+}\right]_{\text {bath }}=14.5$, (d) Seizure-like event at $\left[K^{+}\right]_{\text {bath }}=18.5$, (e) Status epilepticus-like event at $\left[K^{+}\right]_{b a t h}=24.5$, (f) Depolarization block at $\left[K^{+}\right]_{b a t h}=25.5$.

and relative heterogeneity $\Delta / J$. This provides evidence that the mean-field approximation can capture the network behavior of the large network of neurons quite efficiently in that the relative difference between the frequency of the network of neurons and the mean-field approximation is less than $10 \%$ for almost the entire regime of relative heterogeneity and $\left[\mathrm{K}^{+}\right]_{\text {bath }}$.

The simulation of the mean-field model along with the network of single neurons demonstrates that the mean-field approximation is quite robust in terms of the distribution of heterogeneity, as illustrated in Fig. 4(d), with a Gaussian distribution of heterogeneity rather than Lorentzian. Moreover, the simulations of a network of single neurons with stochastic white noise reveal that the mean-field model (deterministic) was in agreement with and approximately matched the network of stochastic single neurons for the small variances associated with white noise Fig. 4(e), but as the variance increased, the agreement was reduced and eventually for higher variances the similarity was destroyed as the network simulations of stochastic neurons became more and more noisy (Fig. 4(f)).

\section{Bifurcation analysis of $\left[\mathrm{K}^{+}\right]_{\text {bath }}$ reveals healthy and epileptic-like regimes}

The simulation of the mean-fields dynamics reveals that $\left[K^{+}\right]_{\text {bath }}$ serves as a bifurcation parameter of the dynamics. Scanning through its values in the biophysical range changes the behavior of the dynamics from a stable fixed point to an oscillatory limit cycle type of behavior and back to a stable fixed point. To carry out the bifurcation analysis, numerical continuation was applied, and we found that a Hopf bifurcation exists at $\left[K^{+}\right]_{b a t h}=7.68$ and a reverse Hopf bifurcation could be found at $\left[\mathrm{K}^{+}\right]_{\text {bath }}=25.02$. In between these two values of $\left[\mathrm{K}^{+}\right]_{\text {bath }}$, the dynamics manifests an oscillatory limit cycle behavior, whereas outside of this range the dynamics of the system shows a stable fixed-point behavior.

Fig. 5 presents the 3-dimensional bifurcation diagram (Fig. 5(a)) in $v$ and $\Delta\left[K^{+}\right]_{i}$ with respect to $\left[K^{+}\right]_{b a t h}$, and the 2-dimensional projections (Fig. 5(b)) in the $v$ dimension for the mean-fields model Eq. (29), indicating the Hopf bifurcation. Multistable behavior of the mean-field was found in this model for $\left[K^{+}\right]_{\text {bath }}>5.971$, as it is demonstrated in Fig. 5. Plots in Fig. 5(c), and Fig. 5(e) illustrates the time series of the dynamics for healthy $\left(\left[K^{+}\right]_{\text {bath }}=7.65\right)$, and bursting $\left(\left[K^{+}\right]_{\text {bath }}=11.5,18.5\right)$ states respectively. whereas Fig. 5(d), and Fig. 5(f) represent the phase space trajectories for corresponding healthy, and bursting states. Together, they indicate the up state, down state, and epileptic state for different values of $\left[K^{+}\right]_{\text {bath }}$. When $\left[\mathrm{K}^{+}\right]_{\text {bath }}$ is smaller than the Hopf bifurcation, the downstate is found to be a stable fixed-point, which destabilizes into spiking, and bursting behaviors and gradually into epileptic bursts at the bifurcation. On the other hand, the upstate emerges at $\left[K^{+}\right]_{\text {bath }}=5.971$, which is a stable fixed point of the five-dimensional system (Eq. (28), and Eq. (29)). However, if the fast 
bioRxiv preprint doi: https://doi.org/10.1101/2021.10.29.466427; this version posted December 7, 2021. The copyright holder for this preprint (which was not certified by peer review) is the author/funder, who has granted bioRxiv a license to display the preprint in perpetuity. It is made Biophysical neural mass model of ion-exchange available under aCC-BY-NC-ND 4.0 International license.

(a) Square wave external stimulus current

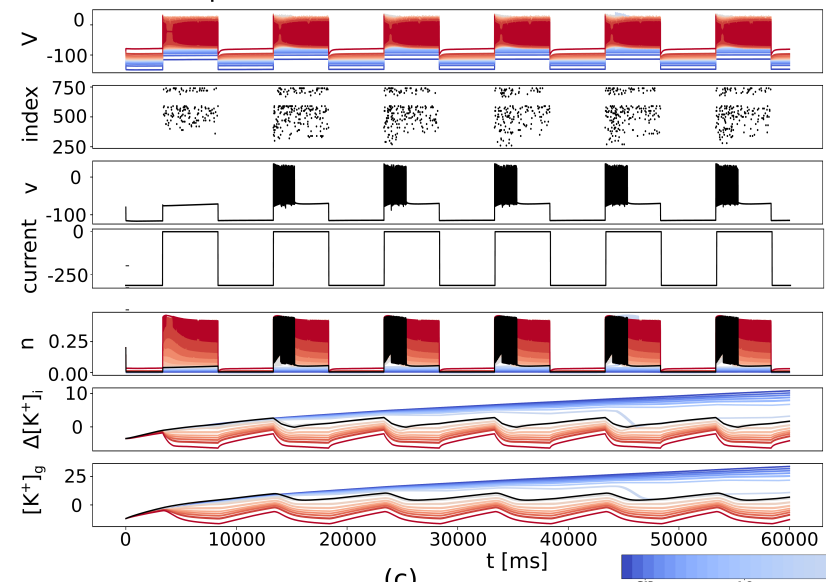

(c)

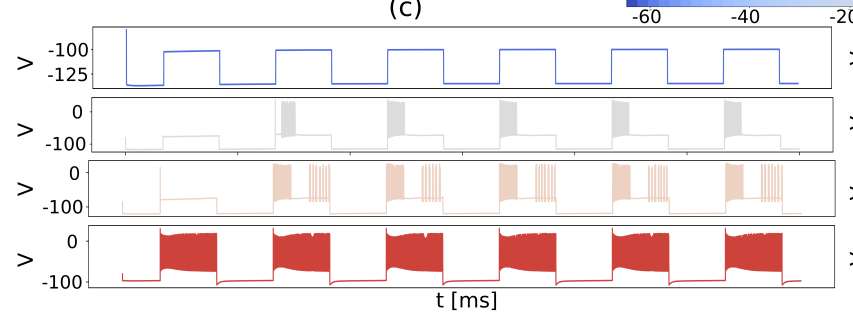

(b) Sinusoidal external stimulus current

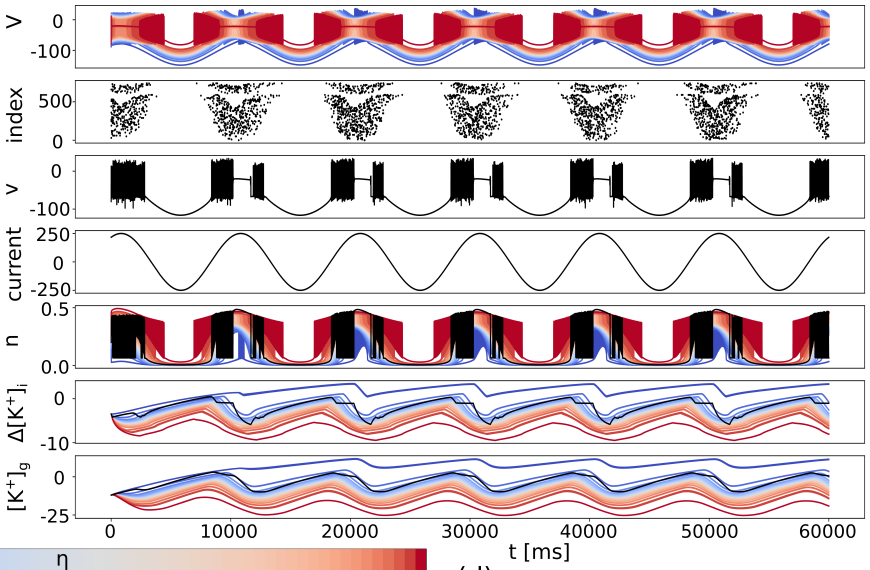

(d)

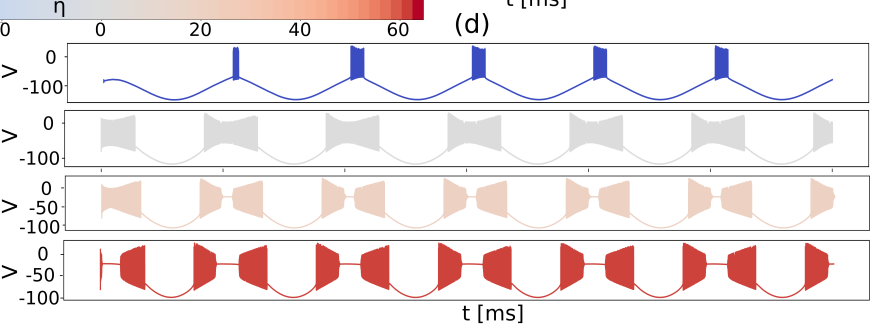

Figure 7. Transition between up and down states with external stimulus current in fixed point (healthy) regime $\left[K^{+}\right]_{\text {bath }}=6.75\left[\mathrm{~mol} \backslash \mathrm{m}^{3}\right]$. Simulations of networks of neurons with the mean-field model: $V$ [mV] for the network nodes in the first row; scatter of the firing neurons in the second row; $v[\mathrm{mV}]$ for the mean-field in the third row; the external input current $[\mathrm{mA}]$ (homogeneous for each node and the same external current is added to the mean-field model) in the fourth row; $n$ in the fifth row; $\Delta\left[K^{+}\right]_{i}\left[\mathrm{~mol} \backslash \mathrm{m}^{3}\right]$ in the sixth row, and $\left[K^{+}\right]_{g}\left[\mathrm{~mol} \backslash \mathrm{m}^{3}\right]$ in the seventh row. The color of the variables in the network of neuron are sorted according to the heterogeneous component $\eta$ (shown in the color bar) and variables from the mean-field model are plotted in black. Effect of addition of an external stimulus current is shown for (a) square wave type external current, (b) sinusoidal type external current. $V[\mathrm{mV}]$ from some representative network nodes are plotted in last four rows for external stimulus currents (c) square wave type external current, (d) sinusoidal type external current.

subsystem is decoupled and the slow variables are considered to be parameters, the fast subsystem in the upstate undergoes bifurcation, demonstrating a limit cycle, a stable spiral, and a stable fixed point as the value of the slow variable decreases.

The model can also demonstrate different neural activities, as demonstrated in Fig. 6. For different regimes of $\left[K^{+}\right]$bath the mean-field model is capable of producing a large set of brain activities, such as a spike train (Fig. 6(a)), tonic spiking (Fig. 6(b)), bursting (Fig. 6(c)), a seizure-like event (Fig. 6(d)), a status epilepticus -like event (Fig. 6(e)), and a polarization block (Fig. 6(f)).

\section{External stimulus triggers transition between regimes of low and high firing rates}

The effect of a external stimulus applied as homogeneous current for each network node is shown in Fig. 7 for network in fixed point regime (healthy state) at $\left[K^{+}\right]_{\text {bath }}=6.75$. All the relevant dynamics are shown for the microscopic network behavior, which is well captured by the mean-field model. For better understanding of the link to the spiking neurons, the voltage $V$ for some of the network nodes sorted according to the heterogeneous component $\eta$ is also presented. It is observed that due to the external stimulus the electrical behavior of the neuronal network could switch between up and down states and could emit transient bursts even in the healthy regime. These results are in agreement with experimental results of brain stimulation. Emission of transient bursts are shown for external stimulus current with square wave type external current (Fig. 7(a)) and sinusoidal type external current (Fig. 7(b)). The membrane potential from some representative network nodes are shown for some external stimulus currents (square wave type external current Fig. 7(c), sinusoidal type external current Fig. 7(d)). These represents different states in the network of homogeneous $\mathrm{HH}$ neurons and the agreement of the states in $\mathrm{HH}$ neuron with mean input $(\eta)$ and the mean-field model. 
(a) Healthy group of neurons $\left[\mathrm{K}^{+}\right]_{\text {bath }}=5.5$

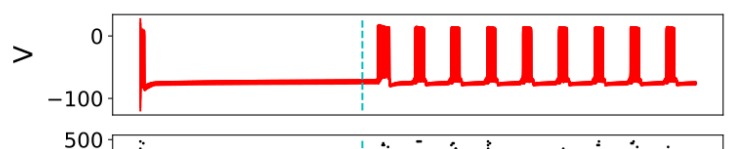

$\begin{array}{rr}\stackrel{\times}{0} & 500 \\ \stackrel{0}{O} & \\ .\end{array}$

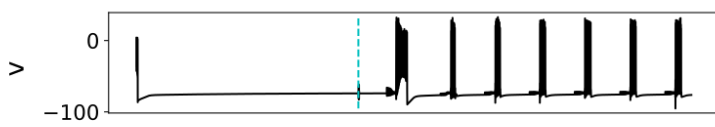

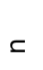
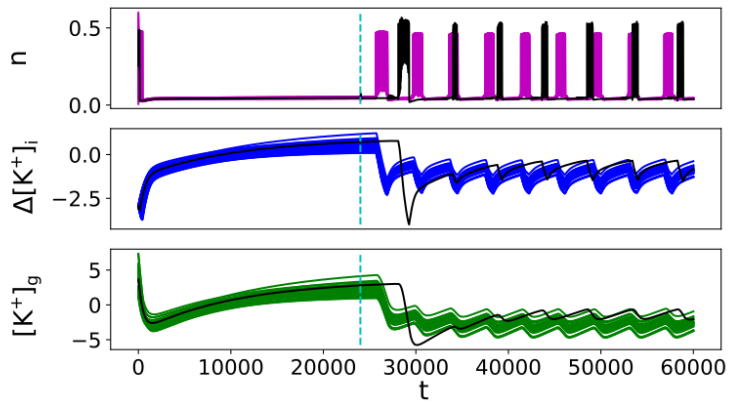

(b) Bursting group of neurons $\left[\mathrm{K}^{+}\right]_{\text {bath }}=11.5$
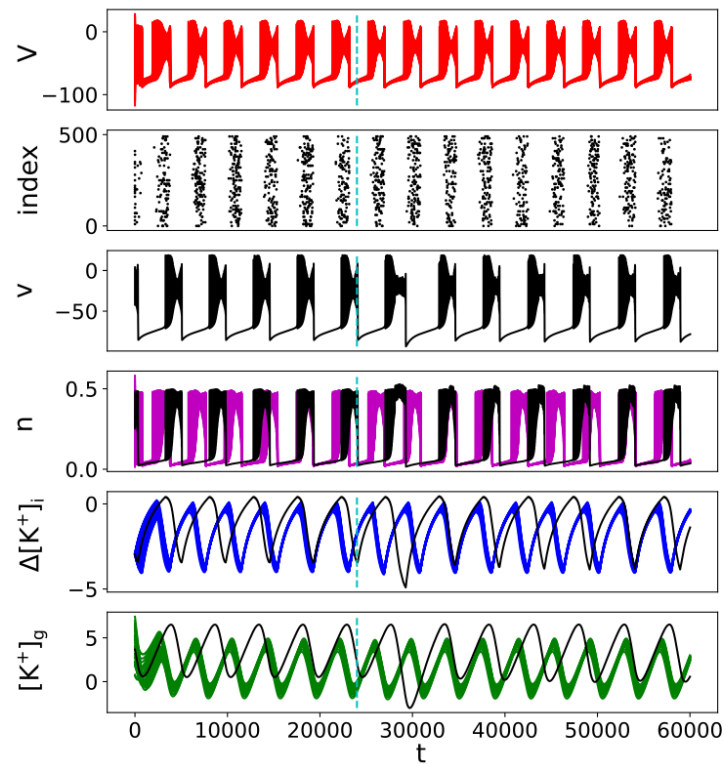

Figure 8. Two coupled mean-field model with the corresponding networks of neurons: $V[\mathrm{mV}]$ for the network nodes in the first row (in red); scatter of the firing neurons in the second row; $v[\mathrm{mV}]$ for the mean-field model in the third row (in black); $n$ in the fourth row: network nodes in magenta and mean-field in black; $\Delta\left[K^{+}\right]_{i}\left[\mathrm{~mol} \backslash \mathrm{m}^{3}\right]$ in the fifth row: network nodes in blue and mean-field in black, and $\left[\mathrm{K}^{+}\right]_{g}\left[\mathrm{~mol} \backslash \mathrm{m}^{3}\right]$ in the sixth row: network nodes in green and mean-field in black, for (a) healthy state (resting state): $\left[K^{+}\right]_{\text {bath }}=5.5$, and (b) bursting state: $\left[K^{+}\right]_{\text {bath }}=11.5$. Initially two populations were uncoupled and at some point of time (denoted by the cyan dotted line) and onward the conducted based coupling was established between them in order to demonstrate two different mode of behaviors, uncoupled and coupled.

\section{Bursting propagation between coupled populations}

The behavior of the mean-field model is demonstrated and validated with the corresponding networks of neurons for the case of two populations coupled with conductance-based coupling, as shown in In Fig. 8. Prior to the coupling (denoted by the cyan dotted line), both populations exhibit different dynamics due to the different values of $\left[\mathrm{K}^{+}\right]_{\text {bath }}$. Once they are coupled, we observed that an otherwise resting state population could be entrained into bursting due to its coupling with a bursting population. This is a common scenario in modelling initiation and propagation of epileptic activity (Jirsa et al., 2017, Proix et al., 2014, Proix et al., 2017, Olmi et al., 2019), and this result could serve as a baseline to explain how neural signals as well as epileptic seizures could propagate between different brain regions.

\section{Discussion}

The general quest of modern neuroscience is understanding and explaining the mechanisms of different brain activities like perception, memory and decision making; but also more generally how the brain functions for healthy individuals during rest, and how it deviates from its healthy state in case of different diseases and disorders. Interestingly most of these brain activities are accessed through measurements of brain dynamics that manifest the collective electrical activities of a huge population of neurons demonstrating some inter-related electrical behavior in different brain regions. Therefore, the brain activities in general can not be explained in microscopic level that is as the mechanism of a single neuron rather they emerge in macroscopic level as a result of the interaction between large population of neurons. Also, besides the recent progress (Markram et al., 2015), it is still not possible to compute the behavior of a few billions of such neurons (which comprises a mammalian brain), and even if it was, it remains questionable what knowledge would we gain from such a complex system (Frégnac, 2017). Hence, the most realistic way to model the brain activities is to group a number of neurons together and write the mathematical descriptions for a group of neurons or large population (Sanz-Leon et al., 2015), using formalism from statistical physics and mathematics. In this case we measure some property of the whole population, such as the mean membrane potential, rather than the activity of an individual neuron. Some of these properties do not even exist for an individual component, for example temperature or 
pressure in case of molecular ensembles, order parameter (Kuramoto, 1984) and mean ensemble frequency (Petkoski et al., 2013) for coupled oscillators, or more specifically the firing rate in case of neuronal population (Gerstner and Kistler, 2002). However, to the best of our knowledge till the date there is no theory that can explain the behavior of large population of neurons (brain areas) from the perspective of the driving mechanism of the neuronal activities, that is the ion-exchange and transportation dynamics in the cellular level. Different pathological trials and experiments have already revealed that changes in ion concentration in brain regions could result in different brain dysfunction but the pathway of these phenomena is still unclear.

In this study we developed a biophysically inspired mean-field model for a network of all-to-all connected, locally homogeneous Hodgkin-Huxley type neurons which are characterized by ion-exchange mechanism across the cellular space. The intermediate approximation into the step wise quadratic function allowed to apply analytical formalism to the complex Hodgkin-Huxley type equations and derive the distribution of membrane potential as Lorentzian distribution. The Lorentzian ansatz makes the mean-field approximation to be analytically tractable, and from the simulation of network behavior of such neurons it is evident that the mean-field model captures the dynamic behavior of the network, while being quite robust for different distributions of heterogeneity and even for noise of small magnitude. This model relates the mechanism of the biophysical activity of ion-exchange and ion channel transportation to the phenomenology of the whole brain dynamics. The major characteristic feature of this model is the analytical bridging between intracellular and extracellular potassium concentrations which in principal act as an adaptation (a biophysical regulation that changes the electrical activity of neurons, acting on a relatively slow time scale), and fast electrical activities in different brain region demonstrated via brain imaging of different modalities. Specifically, the derived model relates the slow scale biophysical mechanism of ion-exchange and transportation in the brain to the fast scale electrical activities of large neuronal ensembles in terms of mean-field formalism of the membrane potential.

Analysing the model, we found the extra-cellular potassium concentration (potassium bath) and heterogeneity of individual neurons in the population to be two significant determinants of the dynamics. Together they take care of the biophysical state of neuronal population (brain region) in terms of ion concentration and structural diversity. In principle these two factors are major drivers of the neuronal dynamics and brain activities and successfully emerged from our simulations. Moreover, the developed model demonstrates the coexistence of resting state brain dynamics and epileptic seizures depends on different states of biophysical quantities and parameters. This aforementioned analytical formalism demonstrates a class of different neural activities, such as the existence of up and down states during the healthy parametric regimes, which is the hallmark of many mean-field representations of linear (Di Volo et al., 2019, Zerlaut et al., 2018) or quadratic integrate and fire neurons (Coombes and Byrne, 2019, Montbrió et al., 2015), and rate models (Wong and Wang, 2006). Increasing the excitability in terms of ion concentration, on the other hand leads to appearance of spike trains, tonic spiking, bursting, seizure-like events, status epilepticus -like events, and depolarization block, similarly as in the epileptor (Jirsa et al., 2014, El Houssaini et al., 2015b, El Houssaini et al., 2020). The mean-field approximation model links the high and low firing rate states (so called up and down state) as well as spiking and bursting behaviors of electrical excitability in neuronal dynamics with the biophysical state of neuronal ensemble (brain regions) in terms of the ion concentrations across the cellular space. The effect of different kind of stimulus current is also analyzed and it is observed that even within the healthy regime, several stimulation, which could either be an external stimuli or some input from some other brain regions, could generate transient spiking and bursting activity in different brain regions. This result is particularly interesting in case of brain stimulation. For example several kind brain stimulation in epileptic patients have already been found to generate epileptic seizures in pathological practice (Fisher and Velasco, 2014, Kahane and Depaulis, 2010). Our results demonstrate that these phenomena could be analytically reproduced and tracked. Therefore, we assume the derived model could potentially be applied to improve predictive capacities in several type of brain disorders, and particularly in epilepsy.

Using conductance-based coupling between two neuronal masses we also demonstrated that a bursting population of neurons can propagate bursting and spiking behavior to an otherwise healthy population. This result is validated by the symmetry in qualitative behavior found in the simulation of the mean-field model and the corresponding network of Hodgkin-Huxley type neurons. This could lead the path to understand how brain signals propagates as a coordinated phenomena depending on the distribution of biophysical quantities and structural as well as architectural heterogeneity on the complex network structure of the connectome. It would also be very interesting to study how this signal propagation varies among healthy subjects and compared with patients with neurological disorders. For an example this model could serve as a computational base line to understand the core question of epilepsy research that is how epileptic seizures propagate from epileptogenic zone to propagation zone and to precisely identify these brain regions in terms of their biophysical states characterized by the distribution of the relevant biophysical and pathological markers as well as structural properties.

Until now, mean-field models used in large scale network simulations like the Virtual Brain did not take into consideration the extracellular space. This model is a first step towards the integration of biophysical processes that may play a key role 
in controlling network behavior. We have started with $K^{+}$, given its known role on neuronal activity. Changes in $K^{+}$occur when the brain alternates between arousal and sleep (Ding et al., 2016). Our model, once integrated in a whole brain model, will allow studying such physiological mechanisms. Although a causal relationship is not clearly established, seizures and spreading depression, which is assumed to underlie certain forms of migraine (Tottene et al., 2009, Vinogradova, 2018), are associated with large $(>6 \mathrm{mM})$ and very large $(>12 \mathrm{mM})$ concentrations of $K^{+}$(Tottene et al., 2009, Hertz and Chen, 2016). The extracellular concentration of $K^{+}$is tightly controlled by astrocytes (Breslin et al., 2018, Nwaobi et al., 2016, Hansson et al., 2000). Most large-scale simulations do not integrate astrocytes, which make half of brain cells (Hansson et al., 2000, Breslin et al., 2018). Their functions are altered in most, if not all, brain disorders, in particular epilepsy (Bedner and Steinhäuser, 2013, Crunelli et al., 2015). Our approach allows formalizing the astrocytic control of extracellular $\mathrm{K}^{+}$. Other ion species also vary during arousal/sleep and seizures, in particular $\mathrm{Ca}^{2+}$ (Ding et al., 2016, Pocock and Kettenmann, 2007, Auld and Robitaille, 2003, Fernandez-Chacon et al., 2001). A decrease in $\mathrm{Ca}^{2+}$ will decrease neurotransmission and thus change cell to cell communication (Pocock and Kettenmann, 2007, Auld and Robitaille, 2003, Fernandez-Chacon et al., 2001). Future studies are needed to integrate $\mathrm{Ca}^{2+}$ in neural mass models (NMM).

Our mean-field derivation aggregates a large class of brain activities and behavior patterns into a single neural mass model, with direct correspondence to biologically relevant parameters. This paves the road for brain network models with bottom-up approach incorporating the regional heterogeneity that stems from the structural data features. We believe that mean-field formalism addressing the biophysical information across neurons will tell us more interesting things about the relation of different dynamics of the brain with its biophysical parameters. This would eventually lead to identifying pathologically measurable bio-markers for large scale brain activities and consequently offering therapeutic targets for different brain dysfunctions.

\section{References}

Amunts K, Mohlberg H, Bludau S, Zilles K (2020) Julich-Brain: A 3D probabilistic atlas of the human brain's cytoarchitecture. Science 369:988-992.

Amzica F, Massimini M, Manfridi A (2002) Spatial buffering during slow and paroxysmal sleep oscillations in cortical networks of glial cells in vivo. Journal of Neuroscience 22:1042-1053.

Ashwin P, Coombes S, Nicks R (2016) Mathematical frameworks for oscillatory network dynamics in neuroscience. The Journal of Mathematical Neuroscience 6:1-92.

Auld DS, Robitaille R (2003) Glial cells and neurotransmission: an inclusive view of synaptic function. Neuron 40:389-400.

Baladron J, Fasoli D, Faugeras O, Touboul J (2012) Mean-field description and propagation of chaos in networks of hodgkin-huxley and fitzhugh-nagumo neurons. The Journal of Mathematical Neuroscience 2:1-50.

Bandyopadhyay A, Kar S (2018) Impact of network structure on synchronization of Hindmarsh-Rose neurons coupled in structured network. Applied Mathematics and Computation 333:194-212.

Baroni F, Burkitt AN, Grayden DB (2014) Interplay of intrinsic and synaptic conductances in the generation of high-frequency oscillations in interneuronal networks with irregular spiking. PLoS Computational Biology 10:e1003574.

Bazhenov M, Timofeev I, Steriade M, Sejnowski TJ (2004) Potassium model for slow (2-3 hz) in vivo neocortical paroxysmal oscillations. Journal of neurophysiology 92:1116-1132.

Bedner P, Steinhäuser C (2013) Altered kir and gap junction channels in temporal lobe epilepsy. Neurochemistry international 63:682-687.

Breslin K, Wade JJ, Wong-Lin K, Harkin J, Flanagan B, Van Zalinge H, Hall S, Walker M, Verkhratsky A, McDaid L (2018) Potassium and sodium microdomains in thin astroglial processes: A computational model study. PLoS computational biology 14:e1006151.

Byrne A, Brookes MJ, Coombes S (2017) A mean field model for movement induced changes in the beta rhythm. Journal of computational neuroscience 43:143-158.

Coombes S, Byrne Á (2019) Next generation neural mass models In Nonlinear dynamics in computational neuroscience, pp. 1-16. Springer.

Courtiol J, Guye M, Bartolomei F, Petkoski S, Jirsa VK (2020) Dynamical mechanisms of interictal resting-state functional connectivity in epilepsy. Journal of Neuroscience 40:5572-5588.

Cressman JR, Ullah G, Ziburkus J, Schiff SJ, Barreto E (2009) The influence of sodium and potassium dynamics on excitability, seizures, and the stability of persistent states: I. single neuron dynamics. Journal of computational neuroscience 26:159-170. 
Crunelli V, Carmignoto G, Steinhäuser C (2015) Novel astrocyte targets: new avenues for the therapeutic treatment of epilepsy. The Neuroscientist 21:62-83.

David O, Friston KJ (2003) A neural mass model for meg/eeg:: coupling and neuronal dynamics. NeuroImage 20:1743-1755.

Deco G, Jirsa VK (2012) Ongoing cortical activity at rest: criticality, multistability, and ghost attractors. Journal of Neuroscience 32:3366-3375.

Deco G, Jirsa VK, McIntosh AR (2011) Emerging concepts for the dynamical organization of resting-state activity in the brain. Nature Reviews Neuroscience 12:43-56.

Deco G, Jirsa VK, Robinson PA, Breakspear M, Friston K (2008) The dynamic brain: from spiking neurons to neural masses and cortical fields. PLoS computational biology 4:e1000092.

Deco G, Kringelbach ML, Arnatkeviciute A, Oldham S, Sabaroedin K, Rogasch NC, Aquino KM, Fornito A (2021) Dynamical consequences of regional heterogeneity in the brain's transcriptional landscape. Science Advances 7.

Depannemaecker D, Ivanov A, Lillo D, Spek L, Bernard C, Jirsa V (2021) A unified physiological framework of transitions between seizures, sustained ictal activity, and depolarization block at the single neuron level. BioRxiv pp. 2020-10.

Destexhe A, Mainen ZF, Sejnowski TJ (1994) An efficient method for computing synaptic conductances based on a kinetic model of receptor binding. Neural computation 6:14-18.

Devalle F, Montbrió E, Pazó D (2018) Dynamics of a large system of spiking neurons with synaptic delay. Physical Review E 98:042214.

Di Volo M, Romagnoni A, Capone C, Destexhe A (2019) Biologically realistic mean-field models of conductance-based networks of spiking neurons with adaptation. Neural computation 31:653-680.

Ding F, O'donnell J, Xu Q, Kang N, Goldman N, Nedergaard M (2016) Changes in the composition of brain interstitial ions control the sleep-wake cycle. Science 352:550-555.

El Houssaini K, Bernard C, Jirsa VK (2020) The epileptor model: a systematic mathematical analysis linked to the dynamics of seizures, refractory status epilepticus, and depolarization block. Eneuro 7.

El Houssaini K, Ivanov AI, Bernard C, Jirsa VK (2015a) Seizures, refractory status epilepticus, and depolarization block as endogenous brain activities. Physical Review E 91:010701.

El Houssaini K, Ivanov AI, Bernard C, Jirsa VK (2015b) Seizures, refractory status epilepticus, and depolarization block as endogenous brain activities. Physical Review E - Statistical, Nonlinear, and Soft Matter Physics 91:2-6.

Fernandez-Chacon R, Königstorfer A, Gerber SH, García J, Matos MF, Stevens CF, Brose N, Rizo J, Rosenmund C, Südhof TC (2001) Synaptotagmin i functions as a calcium regulator of release probability. Nature 410:41-49.

Fisher RS, Velasco AL (2014) Electrical brain stimulation for epilepsy. Nature Reviews Neurology 10:261-270.

Frégnac Y (2017) Big data and the industrialization of neuroscience: A safe roadmap for understanding the brain? Science 358:470-477.

Fröhlich F, Timofeev I, Sejnowski TJ, Bazhenov M (2008) Extracellular potassium dynamics and epileptogenesis In Computational neuroscience in epilepsy, pp. 419-439. Elsevier.

Gerstner W, Kistler WM (2002) Spiking neuron models: Single neurons, populations, plasticity Cambridge university press.

Hansson E, Muyderman H, Leonova J, Allansson L, Sinclair J, Blomstrand F, Thorlin T, Nilsson M, Rönnbäck L (2000) Astroglia and glutamate in physiology and pathology: aspects on glutamate transport, glutamate-induced cell swelling and gap-junction communication. Neurochemistry international 37:317-329.

Hertz L, Chen Y (2016) Importance of astrocytes for potassium ion (k+) homeostasis in brain and glial effects of $k+$ and its transporters on learning. Neuroscience \& Biobehavioral Reviews 71:484-505.

Hocepied G, Legros B, Van Bogaert P, Grenez F, Nonclercq A (2013) Early detection of epileptic seizures based on parameter identification of neural mass model. Computers in biology and medicine 43:1773-1782.

Hodgkin AL, Huxley AF (1952) A quantitative description of membrane current and its application to conduction and excitation in nerve. The Journal of physiology 117:500-544.

Izhikevich EM, Edelman GM (2008) Large-scale model of mammalian thalamocortical systems. Proceedings of the national academy of sciences 105:3593-3598.

Jirsa VK, Proix T, Perdikis D, Woodman MM, Wang H, Gonzalez-Martinez J, Bernard C, Bénar C, Guye M, Chauvel P et al. (2017) The virtual epileptic patient: individualized whole-brain models of epilepsy spread. Neuroimage 145:377-388. 
Jirsa VK, Stacey WC, Quilichini PP, Ivanov AI, Bernard C (2014) On the nature of seizure dynamics. Brain 137:2210-2230. Kahane P, Depaulis A (2010) Deep brain stimulation in epilepsy: what is next? Current opinion in neurology 23:177-182.

Kuramoto Y (1984) Chemical Oscillations, Waves, and Turbulence, Vol. 19 of Springer Series in Synergetics Springer Berlin Heidelberg.

Kuramoto Y (1991) Collective synchronization of pulse-coupled oscillators and excitable units. Physica D: Nonlinear Phenomena 50:15-30.

Laing CR (2017) Phase oscillator network models of brain dynamics. Computational models of brain and behavior 505:517.

Luke TB, Barreto E, So P (2013) Complete classification of the macroscopic behavior of a heterogeneous network of theta neurons. Neural computation 25:3207-3234.

Markram H, Muller E, Ramaswamy S, Reimann MW, Abdellah M, Sanchez CA, Ailamaki A, Alonso-Nanclares L, Antille N, Arsever S, Kahou GAA, Berger TK, Bilgili A, Buncic N, Chalimourda A, Chindemi G, Courcol JD, Delalondre F, Delattre V, Druckmann S, Dumusc R, Dynes J, Eilemann S, Gal E, Gevaert ME, Ghobril JP, Gidon A, Graham JW, Gupta A, Haenel V, Hay E, Heinis T, Hernando JB, Hines M, Kanari L, Keller D, Kenyon J, Khazen G, Kim Y, King JG, Kisvarday Z, Kumbhar P, Lasserre S, Le Bé JV, Magalhães BRC, Merchán-Pérez A, Meystre J, Morrice BR, Muller J, Muñoz-Céspedes A, Muralidhar S, Muthurasa K, Nachbaur D, Newton TH, Nolte M, Ovcharenko A, Palacios J, Pastor L, Perin R, Ranjan R, Riachi I, Rodríguez JR, Riquelme JL, Rössert C, Sfyrakis K, Shi Y, Shillcock JC, Silberberg G, Silva R, Tauheed F, Telefont M, Toledo-Rodriguez M, Tränkler T, Van Geit W, Díaz JV, Walker R, Wang Y, Zaninetta SM, Defelipe J, Hill SL, Segev I, Schürmann F (2015) Reconstruction and Simulation of Neocortical Microcircuitry. Cell 163:456-492.

Melozzi F, Bergmann E, Harris JA, Kahn I, Jirsa V, Bernard C (2019) Individual structural features constrain the mouse functional connectome. Proceedings of the National Academy of Sciences of the United States of America 116:26961-26969.

Montbrió E, Pazó D (2020) Exact mean-field theory explains the dual role of electrical synapses in collective synchronization. Physical Review Letters 125:248101.

Montbrió E, Pazó D, Roxin A (2015) Macroscopic description for networks of spiking neurons. Physical Review X 5:021028.

Moran RJ, Kiebel SJ, Stephan KE, Reilly R, Daunizeau J, Friston KJ (2007) A neural mass model of spectral responses in electrophysiology. NeuroImage 37:706-720.

Nevado-Holgado AJ, Marten F, Richardson MP, Terry JR (2012) Characterising the dynamics of eeg waveforms as the path through parameter space of a neural mass model: application to epilepsy seizure evolution. Neuroimage 59:2374-2392.

Nwaobi SE, Cuddapah VA, Patterson KC, Randolph AC, Olsen ML (2016) The role of glial-specific kir4. 1 in normal and pathological states of the cns. Acta neuropathologica 132:1-21.

Olmi S, Petkoski S, Guye M, Bartolomei F, Jirsa V (2019) Controlling seizure propagation in large-scale brain networks. PLoS Computational Biology 15:e1006805.

Park EH, Durand DM (2006) Role of potassium lateral diffusion in non-synaptic epilepsy: a computational study. Journal of theoretical biology 238:666-682.

Petkoski S, Iatsenko D, Basnarkov L, Stefanovska A (2013) Mean-field and mean-ensemble frequencies of a system of coupled oscillators. Physical Review E - Statistical, Nonlinear, and Soft Matter Physics 87:1-12.

Petkoski S, Jirsa VK (2019) Transmission time delays organize the brain network synchronization. Philosophical Transactions of the Royal Society A: Mathematical, Physical and Engineering Sciences 377:20180132.

Petkoski S, Stefanovska A (2012) Kuramoto model with time-varying parameters. Physical Review E 86:046212.

Pocock JM, Kettenmann H (2007) Neurotransmitter receptors on microglia. Trends in neurosciences 30:527-535.

Proix T, Bartolomei F, Chauvel P, Bernard C, Jirsa VK (2014) Permittivity coupling across brain regions determines seizure recruitment in partial epilepsy. Journal of Neuroscience 34:15009-15021.

Proix T, Bartolomei F, Guye M, Jirsa VK (2017) Individual brain structure and modelling predict seizure propagation. Brain 140:641-654.

Ransom CB, Ransom BR, Sontheimer H (2000) Activity-dependent extracellular k+ accumulation in rat optic nerve: the role of glial and axonal nat pumps. The Journal of physiology 522:427-442.

Roxin A, Brunel N, Hansel D (2005) Role of delays in shaping spatiotemporal dynamics of neuronal activity in large networks. Physical review letters 94:238103. 
Sanz-Leon P, Knock SA, Spiegler A, Jirsa VK (2015) Mathematical framework for large-scale brain network modeling in the virtual brain. Neuroimage 111:385-430.

Shriki O, Hansel D, Sompolinsky H (2003) Rate models for conductance-based cortical neuronal networks. Neural computation 15:1809-1841.

Spiegler A, Knösche TR, Schwab K, Haueisen J, Atay FM (2011) Modeling brain resonance phenomena using a neural mass model. PLoS computational biology 7:e1002298.

Tottene A, Conti R, Fabbro A, Vecchia D, Shapovalova M, Santello M, van den Maagdenberg AM, Ferrari MD, Pietrobon D (2009) Enhanced excitatory transmission at cortical synapses as the basis for facilitated spreading depression in cav2. 1 knockin migraine mice. Neuron 61:762-773.

Touboul J, Wendling F, Chauvel P, Faugeras O (2011) Neural mass activity, bifurcations, and epilepsy. Neural computation 23:3232-3286.

Ullah G, Cressman Jr JR, Barreto E, Schiff SJ (2009) The influence of sodium and potassium dynamics on excitability, seizures, and the stability of persistent states: Ii. network and glial dynamics. Journal of computational neuroscience 26:171-183.

Vinogradova LV (2018) Initiation of spreading depression by synaptic and network hyperactivity: Insights into trigger mechanisms of migraine aura. Cephalalgia 38:1177-1187.

Wendling F, Benquet P, Bartolomei F, Jirsa V (2016) Computational models of epileptiform activity. Journal of neuroscience methods 260:233-251.

Wilson HR, Cowan JD (1972) Excitatory and inhibitory interactions in localized populations of model neurons. Biophysical journal 12:1-24.

Wong KF, Wang XJ (2006) A recurrent network mechanism of time integration in perceptual decisions. Journal of Neuroscience 26:1314-1328.

Zerlaut Y, Chemla S, Chavane F, Destexhe A (2018) Modeling mesoscopic cortical dynamics using a mean-field model of conductance-based networks of adaptive exponential integrate-and-fire neurons. Journal of computational neuroscience 44:45-61.

\section{Author contributions}

A.B., S.P., and V.K.J. designed research; A.B., S.P., and V.K.J. performed research; A.B. simulated the models and analyzed the results; A.B., C.B., S.P., and V.K.J. wrote the paper. *S.P. and *V.K.J. contributed equally to this work as last author. The authors declare no competing financial interests. Correspondence should be addressed to Spase Petkoski at spase.petkoski@univ-amu.fr or Viktor K. Jirsa at viktor.jirsa@univ-amu.fr.

\section{Acknowledgments}

This research was supported by the European Union's Horizon 2020 research and innovation programme under grant agreement No. 945539 (SGA3) Human Brain Project. CB received support from the Agence nationale de la recherche projects ANR-17CE37-0001-01 and ANR-20-NEUC-0005-02. 